\title{
EXAMPLE OF MINIMIZER OF THE AVERAGE-DISTANCE PROBLEM WITH NON CLOSED SET OF CORNERS
}

\author{
XIN YANG LU
}

ABSTRACT. The average-distance problem, in the penalized formulation, involves minimizing

$$
E_{\mu}^{\lambda}(\Sigma):=\int_{\mathbb{R}^{d}} \inf _{y \in \Sigma}|x-y| d \mu(x)+\lambda \mathcal{H}^{1}(\Sigma)
$$

among path-wise connected, closed sets $\Sigma$ with finite $\mathcal{H}^{1}$-measure, where $d \geq 2, \mu$ is a given measure and $\lambda$ a given parameter. Regularity of minimizers is a delicate problem. It is known that even if $\mu \ll \mathcal{L}^{d}, C^{1}$ regularity does not hold in general. An interesting question is whether the set of corners, i.e. points where $C^{1}$ regularity does not hold, is closed. The aim of this paper is to provide an example of minimizer whose set of corners is not closed, with reference measure $\mu$ absolutely continuous with respect to Lebesgue measure.

Keywords. nonlocal variational problem, average-distance problem, regularity Classification. 49Q20, 49K10, 49Q10, 35B65

\section{INTRODUCTION}

The average-distance problem, in the penalized formulation, was introduced by Buttazzo, Mainini and Stepanov in [1]:

Problem 1.1. Given $d \geq 2$ a measure $\mu$, and a parameter $\lambda>0$, minimize

$$
E_{\mu}^{\lambda}: \mathcal{A} \longrightarrow \mathbb{R}, \quad E_{\mu}^{\lambda}(\Sigma):=F_{\mu}(\Sigma)+\lambda \mathcal{H}^{1}(\Sigma),
$$

where

$$
F_{\mu}: \mathcal{A} \longrightarrow \mathbb{R}, \quad F_{\mu}(\Sigma):=\int_{\mathbb{R}^{d}} d(x, \Sigma) d \mu
$$

$$
\mathcal{A}:=\left\{\Sigma \subseteq \mathbb{R}^{d}: \Sigma \text { compact, path-wise connected, } \mathcal{H}^{1}(\Sigma)<\infty\right\},
$$

$d(x, \Sigma):=d_{\mathcal{H}}(\{x\}, \Sigma)$ and $d_{\mathcal{H}}$ denotes the Hausdorff distance.

Existence of minimizers follows (see for instance [1, 2, 3]) from Blaschke selection theorem and Gotab theorem. The functional $F_{\mu}$ will be often referred as "average-distance functional", and Problem 1.1 as "average-distance problem". In the following, any considered measure will be assumed nonnegative, compactly supported, probability measures. The choice to work with probability measures is done for the sake of simplicity, and it is not restrictive since results proven in this paper can be easily extended to finite measures.

The average-distance problem originally stemmed from mathematical modeling of optimization problems. A classic application is found in urban planning: let

- $\mu$ be the distribution of passengers in a given region, 
- $\Sigma$ (the unknown) be the transport network to be built.

In this case $F_{\mu}(\Sigma)$ is the "average distance" of passengers from the network (thus smaller values of $F_{\mu}(\Sigma)$ means that $\Sigma$ is "easily accessible"), and $\lambda \mathcal{H}^{1}(\Sigma)$ is the cost to build such network. Thus minimizing $E_{\mu}^{\lambda}$ is determining the network which "best serves" the passengers, under cost considerations.

A recent application is found in data approximation: let

- $\mu$ be the distribution of data points,

- $\Sigma$ (the unknown) be a one dimension set which approximates the data.

In this case $F_{\mu}(\Sigma)$ is the approximation error, while $\lambda \mathcal{H}^{1}(\Sigma)$ is the cost associated to its complexity. Thus minimizing $E_{\mu}^{\lambda}$ is determining the "best" approximation, which balances approximation error and cost. In data approximation the regularity of $\Sigma$ is important: indeed it has been proven (Slepčev [12]) that a positive amount of mass is projected on any point for which $C^{1}$ regularity fails. This corresponds to a loss of information, and it is undesirable.

Regularity of minimizers is quite a delicate problem. It is known that minimizers are finite union (Slepčev et al. [10], Lemma 3.1) of Lipschitz curves (Buttazzo, Oudet, Paolini, Stepanov [2, 3, 11]), but even when $\mu \ll \mathcal{L}^{d}, C^{1}$ regularity is not true in general (Slepčev [12]). However a curvature estimate still holds (Slepčev et al. [10]).

For future reference, given $\Sigma \in \mathcal{A}$, a point $p \in \Sigma$ of degree two (i.e. $\Sigma \backslash\{p\}$ has exactly two connected components, see Definition 2.3 for which $C^{1}$ regularity fails will be referred as "corner". Since the approach used in [12] is only suited for constructing minimizers with finitely many corners, it is unclear if (for minimizers) the set of corners is generally closed, or even finite. The aim of this paper is to provide an example of minimizer whose set of corners is not closed.

This paper will be structured as follows:

- in Section 2 we will recall preliminary results,

- in Section 3 we will construct an explicit example of minimizer of Problem 1.1 whose set of corners is not closed.

\section{PRELIMINARY RESUlTS}

The main goal of this section is to introduce some notations and recall well known results which will be used in Section 3. The average-distance functional satisfies the following well known properties:

(1) given a measure $\mu$ and $\lambda>0$, the mapping $\Sigma \mapsto E_{\mu}^{\lambda}(\Sigma)$ is lower semicontinuous w.r.t. $d_{\mathcal{H}}$

(2) given $\Sigma \in \mathcal{A}$ and $\lambda>0$, the mapping $\mu \mapsto E_{\mu}^{\lambda}(\Sigma)$ is continuous w.r.t. weak ${ }^{*}$ convergence of measures,

(3) if $\left\{\mu_{n}\right\} \stackrel{*}{\rightarrow} \mu$, then for any $\lambda>0$, it holds $E_{\mu_{n}}^{\lambda} \stackrel{\Gamma}{\rightarrow} E_{\mu}^{\lambda}$,

(4) consider a sequence $\left\{\mu_{n}\right\}{ }^{*} \rightarrow \mu$ and for any $n$ choose $\Sigma_{n} \in \operatorname{argmin} E_{\mu_{n}}^{\lambda}$. Then there exists $\Sigma \in \operatorname{argmin} E_{\mu}^{\lambda}$ such that (upon subsequence) $\left\{\Sigma_{n}\right\} \stackrel{d_{\mathcal{H}}}{\rightarrow} \Sigma$.

For further details (including proofs), we refer to [2, 3, 4, 12]. 
Recall that given a set of points $\Pi:=\left\{P_{1}, \cdots, P_{j}\right\} \subseteq \mathbb{R}^{d}$, the Steiner graph of $\Pi$ is a path-wise connected set with minimal length containing $\Pi$. The next result proves an intrinsic connection between Steiner graphs and minimizers of the average distance functional.

Proposition 2.1. Given a discrete probability measure $\mu:=\sum_{i=1}^{n} a_{i} \delta_{x_{i}}$ on $\mathbb{R}^{d}$, with $a_{1}, \cdots, a_{n} \geq 0$ and $\delta$ denoting the Dirac measure supported on the subscripted point, a parameter $\lambda>0$, then any minimizer $\Sigma \in \operatorname{argmin}_{\mathcal{A}} E_{\mu}^{\lambda}$ is a Steiner graph.

Proof. For the proof we refer to [12].

Definition 2.2. Given a discrete probability measure $\mu:=\sum_{i=1}^{n} a_{i} \delta_{x_{i}}$ on $\mathbb{R}^{d}, \lambda>0$, and a minimizer $\Sigma \in \operatorname{argmin}_{\mathcal{A}} E_{\mu}^{\lambda}$, a point $v \in \Sigma$ is a "vertex" if there exists $x \in \operatorname{supp}(\mu)$ such that $d(x, \Sigma)=|x-v|$.

Next we define the notion of "degree" of a point.

Definition 2.3. Given $\Sigma \in \mathcal{A}$, consider a point $v \in \Sigma$ such that $\Sigma \backslash\{v\}$ has finitely many connected components. Then the "degree" of $v$ is defined as the number of connected components of $\Sigma \backslash\{v\}$.

Note that the degree of a $v$ depends also on $\Sigma$. However for the sake of brevity we will omit writing such dependency if no risk of confusion arises. Moreover we recall that it is possible to define the degree of $v$ even when $\Sigma \backslash\{v\}$ has infinitely many connected components (see Definition 2.2 of [4]), but for our purposes this is not required. For the sake of brevity, in the following given two points $p$ and $q$, the symbol $\llbracket p, q \rrbracket$ will denote the straight segment between $p$ and $q$.

In view of Proposition 2.1. a segment having endpoint in two vertices and containing no other vertices will be referred as "edge". The following classic result (see for instance [5, 6]) proves several geometric properties about Steiner graphs:

Proposition 2.4. Given a Steiner graph $G$, it holds:

- $G$ is a tree,

- if $\llbracket u, v \rrbracket$ and $\llbracket v, w \rrbracket$ are edges, with a common vertex $v$, then $\widehat{u v w} \geq 2 \pi / 3$,

- the maximal degree of any vertex is 3 ,

- if $v$ is a vertex of degree 3 , denoting by $\llbracket u_{i}, v \rrbracket, i=1,2,3$ the 3 different edges containing $v$, then the angle between any two such edges is $2 \pi / 3$, and these edges are coplanar.

As done in [12], in view of Propositions 2.1] and 2.4, the following definition will be useful:

Definition 2.5. Given a discrete measure $\mu$, a parameters $\lambda>0$, and $\Sigma \in \operatorname{argmin}_{\mathcal{A}} E_{\mu}^{\lambda}$, a vertex $v \in \Sigma$ is called:

- "endpoint" if has degree 1,

- "corner point" if has degree 2,

- "triple junction" if has degree 3.

If $v$ is a corner point, denoting by $w, z$ the two vertices for which $\llbracket w, v \rrbracket$ and $\llbracket v, z \rrbracket$ are edges, the "turning angle" in $v$ is defined as:

$$
\mathrm{TA}(v):=\pi-\widehat{w v z} .
$$

Similarly, given a subset $A \subseteq \Sigma$, the turning angle of $A$ is defined as

$$
T A(A):=\sum_{u \in A, \text { u corner point }} T A(u) .
$$


Recall that the turning angle TA $(v)$ describes the curvature of $\Sigma$ at $v$. Lemma 3.7 proves that (in our case) corner points coincide with corners (defined as points where $C^{1}$ regularity does not hold). Thus in the following we will identify corner points with corners. Given a discrete measure $\mu$ and $\Sigma \in \mathcal{A}$, for the sake of brevity, the following expressions will be used. Here $v \in \Sigma$, while $x$ is a generic point.

- " $v$ is tied to $x$ ": the vertex $v$ coincides with some point $x \in \operatorname{supp}(\mu)$,

- " $v$ is free": the vertex $v$ coincides with no point $x \in \operatorname{supp}(\mu)$,

- " $x$ talks to $v$ ", " $x$ projects on $v$ ", " $v$ talks to $x$ ": all these mean $d(x, \Sigma)=|x-v|$,

- " $v$ talks to some mass", "mass talking to $v$ ": $v$ talks to some point in $\operatorname{supp}(\mu)$,

- $T M(\mu, v, \Sigma)(T M(v)$ when there is no risk of confusion) denotes the total mass of projecting on $v$. For a detailed discussion see Lemma 2.1 of [10].

- " $H$ mass projects on $v$ ", where $H \geq 0$ : this means $T M(\mu, v, \Sigma)=H$.

The last three expressions will be used even for non discrete measures $\mu$.

The following conditions are the main tools used to analyze minimizers, when the reference measure is discrete.

Proposition 2.6. Given a discrete measure $\mu$, a parameter $\lambda>0$, and $\Sigma \in \operatorname{argmin}_{\mathcal{A}} E_{\mu}^{\lambda}$, it holds:

(1) if $v \in \Sigma$ is a triple junction, then $T M(\mu, v, \Sigma)=0$,

(2) if some point $y \in \operatorname{supp}(\mu)$ talks to different vertices $v, v^{\prime}$, then there exist $x, x^{\prime} \in \operatorname{supp}(\mu) \operatorname{such}$ that $v$ is tied to $x$ and $v^{\prime}$ is tied to $x^{\prime}$,

(3) if $v \in \Sigma$ is an endpoint then $T M(\mu, v, \Sigma) \geq \lambda$,

(4) if $v \in \Sigma$ is a corner, denoting by $w, z$ the two vertices such that $\llbracket w, v \rrbracket$ and $\llbracket v, z \rrbracket$ are edges, then

$$
T A(v) \leq \frac{\pi}{2 \lambda} T M(\mu, v, \Sigma)
$$

For the proof we refer to Lemma 9, Corollary 10 and Lemma 11 of [12]. Note that given a subset $A \subseteq \Sigma$, inequality (1) holds for any corner $v \in A$, and summing over all such corners yields

$$
\mathrm{TA}(A) \leq \frac{\pi}{2 \lambda} \sum_{v \in A, v \text { corner }} T M(\mu, v, \Sigma)
$$

If $\Sigma$ is itself a curve, then

$$
\mathrm{TA}(\Sigma) \leq \frac{\pi}{2 \lambda} \sum_{v \text { corner }} T M(\mu, v, \Sigma)
$$

using Proposition 2.6, zero mass projects on triple junctions, thus all the mass projects on endpoints or corners. Denoting by $P_{0}$ and $P_{1}$ the two endpoints of $\Sigma$ (the case $\Sigma$ being a singleton is trivial), it holds

$$
\begin{aligned}
\mathrm{TA}(\Sigma) & \leq \frac{\pi}{2 \lambda} \sum_{v \text { corner }} T M(\mu, v, \Sigma) \\
& \leq \frac{\pi}{2 \lambda}\left(1-T M\left(\mu, P_{0}, \Sigma\right)-T M\left(\mu, P_{1}, \Sigma\right)\right) \\
& \leq \frac{\pi}{2 \lambda}(1-2 \lambda),
\end{aligned}
$$


where the last inequality follows from point (3) of Proposition 2.6

An similar result has been proven (in [10], to which to refer for the proof) for generic measures:

Lemma 2.7. Given a measure $\mu$, a parameter $\lambda>0$ and $\Sigma \in \operatorname{argmin} E_{\mu}^{\lambda}$, for an subset $A \subseteq \Sigma$ ( $A$ can be a singleton) it holds

$$
\sum_{j}\left\|\alpha_{j}^{\prime}\right\|_{T V} \leq \frac{\pi}{2 \lambda} T M(A)
$$

with $T M(A)$ denoting the mass projected on $A$, and $\alpha_{j}:[0,1] \longrightarrow A$ denoting the constant speed parameterizations of branches making $A$.

Finally we recall a classic convergence result:

Lemma 2.8. Given a sequence of curves $\left\{\gamma_{k}\right\}:[0,1] \longrightarrow K$, with $K \subseteq \mathbb{R}^{2}$ a given compact set, satisfying

$$
\sup _{k}\left\|\gamma_{k}^{\prime}\right\|_{B V}<\infty, \quad \sup _{k} \mathcal{H}^{1}\left(\gamma_{k}([0,1])\right)<\infty
$$

then there exists a curve $\gamma:[0,1] \longrightarrow K$, such that (upon subsequence) it holds:

(1) $\left\{\gamma_{k}\right\} \rightarrow \gamma$ in $C^{\alpha}$ for any $\alpha \in[0,1)$,

(2) $\left\{\gamma_{k}^{\prime}\right\} \rightarrow \gamma^{\prime}$ in $L^{p}$ for any $p \in[1, \infty)$,

(3) $\left\{\gamma_{k}^{\prime \prime}\right\} \stackrel{*}{\rightarrow} \gamma^{\prime \prime}$ in the space of signed Borel measures.

For the sake of brevity, we will never relabel subsequences if no risk of confusion arises.

\section{Counterexample}

The aim of this section is to construct an explicit example of minimizer whose set of corners is not closed.

The reference measure will be:

$$
\mu:=\mu_{\text {heavy }}+\mu_{\text {light }}
$$

where

$$
\begin{aligned}
& \mu_{\text {heavy }}:=\frac{1-\eta}{2}\left(\frac{1}{\mathcal{L}^{2}(B((-L, h), \rho))} \mathcal{L}_{\llcorner B((-L, h), \rho)}^{2}\right)+\frac{1-\eta}{2}\left(\frac{1}{\mathcal{L}^{2}(B((L, h), \rho))} \mathcal{L}_{\llcorner B((L, h), \rho)}^{2}\right) \\
&= \frac{1-\eta}{2 \pi \rho^{2}}\left(\mathcal{L}_{\llcorner B((-L, h), \rho)}^{2}+\mathcal{L}_{\llcorner B((L, h), \rho)}^{2}\right), \\
& \mu_{\text {light }}:=\sum_{n=1}^{\infty} \frac{\mathfrak{m}_{n}}{\mathcal{L}^{2}\left(\mathfrak{B}_{n}\right)} \mathcal{L}_{\left\llcorner\mathfrak{B}_{n}\right.}^{2}=\sum_{n=1}^{\infty} \frac{\mathfrak{m}_{n}}{\pi \mathfrak{r}_{n}^{2}} \mathcal{L}_{\left\llcorner\mathfrak{B}_{n}\right.}^{2}, \\
& \mathfrak{B}_{n}:=B\left(\left(\mathfrak{c}_{n}, 0\right), \mathfrak{r}_{n}\right), \mathfrak{c}_{n}:=m^{-n}, \mathfrak{m}_{n}:=m^{-\left(10^{10} n\right) !}, \mathfrak{r}_{n}:=m^{-\left(\left(10^{100} n\right) !\right) !} .
\end{aligned}
$$

Here by construction $\eta=\sum_{n=1}^{\infty} \mathfrak{m}_{n}$ is the total mass of $\mu_{\text {light }}$. By definition $\mu$ depends on several parameters appearing in (4) and (5). Choosing such parameters will be the main aim of subsection 3.1. For the sake of brevity (unless otherwise specified) we omit writing such dependencies. 


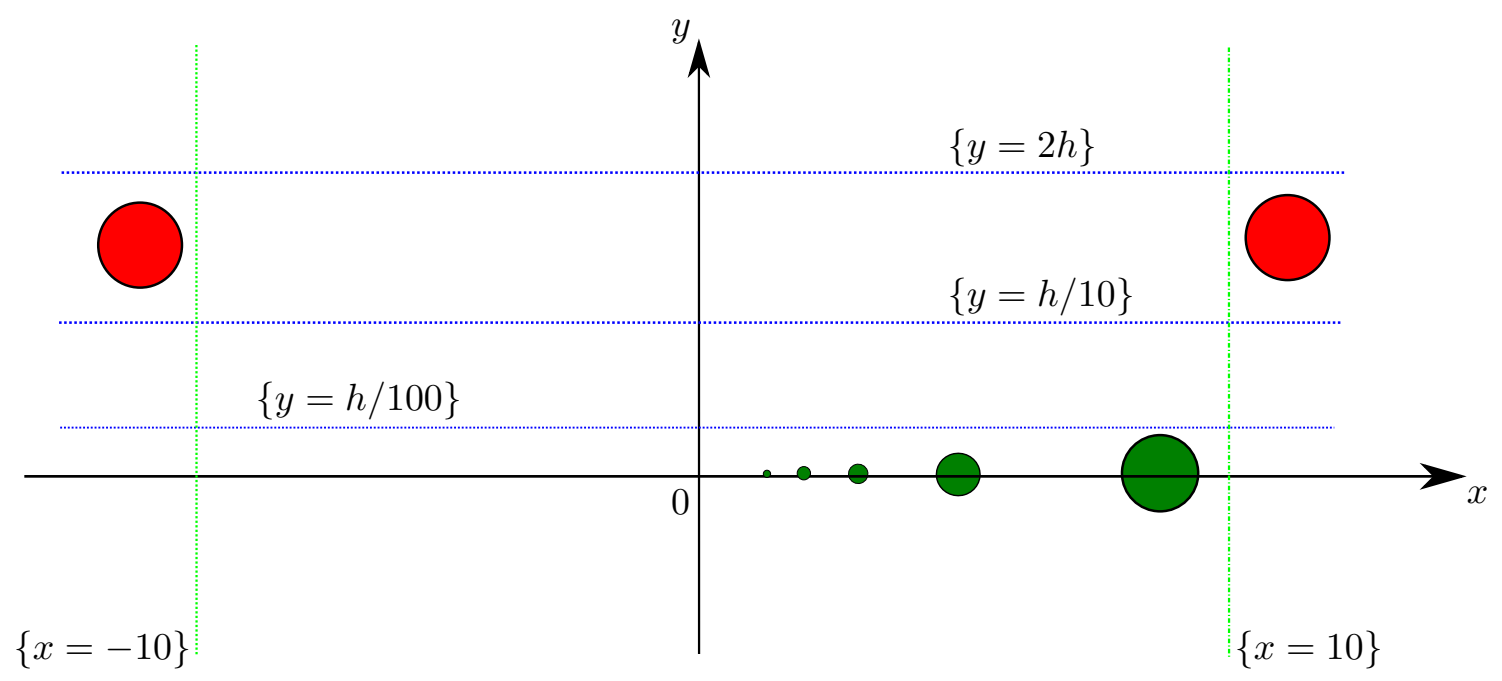

FIGURE 1. This is a representation (highly not to scale) of the supports of $\mu_{\text {heavy }}$ (red) and $\mu_{\text {light }}$ (green). The represented lines will be relevant for our construction.

Intuitively, $\operatorname{supp}\left(\mu_{\text {heavy }}\right)$ is union of two "small, massive and distant" balls, each of which contains "almost one half" of mass; $\operatorname{supp}\left(\mu_{\text {light }}\right)$ is union of balls $\mathfrak{B}_{n}, n \geq 1$, each of which containing mass $\mathfrak{m}_{n}$. As will be clear in the following, $\mu_{\text {light }}$ is the measure that "generates corners", while the role of $\mu_{\text {heavy }}$ is to force minimizers to have "large length" and "little curvature". Note that for $\mu_{\text {light }}$ the distance between two distinct balls $\mathfrak{B}_{n_{1}}$ and $\mathfrak{B}_{n_{2}}$ (assume $n_{2}>n_{1}$ ) is much larger than $\sum_{n=n_{1}}^{n_{2}} \mathfrak{m}_{n}$ (which is roughly "the combined masses of the balls in between"); and for each ball $\mathfrak{B}_{n}$, the mass supported on it $\left(\mathfrak{m}_{n}\right)$ is much larger than its own radius $\left(\mathfrak{r}_{n}\right)$. This will be crucial for our construction.

\subsection{Choosing parameters. Let}

$$
L:=10^{300}, \quad h:=1, \quad m:=10^{100} .
$$

The aim of this subsection is to choose suitable parameters $\lambda$ and $\rho$.

Lemma 3.1. For any $\lambda \in\left(\frac{1-3 \eta}{2}, \frac{1-2 \eta}{2}\right)$ there exists $\rho_{0}>0$ such that for any $\rho \in\left(0, \rho_{0}\right)$, any minimizer of $E_{\mu}^{\lambda}$ is a simple curve with positive length.

Proof. Choose an arbitrary $\lambda \in\left(\frac{1-3 \eta}{2}, \frac{1-2 \eta}{2}\right)$. The proof will be split in two parts.

- Claim 1: any minimizer has at most 2 endpoints.

Proposition 2.6 proves that any minimizer contains at most $[1 / \lambda]$ (here $[\cdot]$ denotes the integer part mapping) endpoints, and hypothesis $\lambda \in\left(\frac{1-3 \eta}{2}, \frac{1-2 \eta}{2}\right)$ implies $[1 / \lambda]<3$, thus the claim is proven.

- Claim 2: for sufficiently small $\rho$, any minimizer of $E_{\mu_{\rho}}^{\lambda}$ has positive length. 
Consider the measure

$$
\mu_{0}:=\mu_{\text {light }}+\frac{1-\eta}{2}\left(\delta_{(-L, h)}+\delta_{(L, h)}\right)
$$

and clearly $\left\{\mu_{\rho}\right\} \stackrel{*}{\rightarrow} \mu_{0}$ as $\rho \rightarrow 0$ (here we highlighted the dependency on $\rho$ ). For any arbitrary point $P:=\left(x_{0}, y_{0}\right)$ it holds

$$
E_{\mu_{0}}^{\lambda}(\{P\}) \geq \frac{1-\eta}{2}(|P-(-L, h)|+|P-(L, h)|) \geq(1-\eta) L .
$$

Let

$$
\Lambda:=\llbracket(-L, h),(L, h) \rrbracket .
$$

It holds (since by hypothesis $2 \lambda<1-\eta$ )

$$
E_{\mu_{0}}^{\lambda}(\Lambda) \leq 2 \lambda L+2 h \eta<(1-\eta) L \leq E_{\mu_{0}}^{\lambda}(\{P\})
$$

Thus any minimizer of $E_{\mu_{0}}^{\lambda}$ has positive length.

Since $\left\{\mu_{\rho}\right\} \stackrel{*}{\rightarrow} \mu_{0}$ as $\rho \rightarrow 0$, for sequences $\left\{\rho_{n}\right\} \rightarrow 0,\left\{\Sigma_{n} \in \operatorname{argmin} E_{\mu_{\rho_{n}}}^{\lambda}\right.$, there exists $\Sigma_{\infty} \in$ $\operatorname{argmin} E_{\mu_{0}}^{\lambda}$ such that (upon subsequence) $\left\{\Sigma_{n}\right\} \stackrel{d_{\mathcal{H}}}{\rightarrow} \Sigma_{\infty}$, and we just proved that such a $\Sigma_{\infty}$ has positive length. Thus the proof is complete.

Lemma 3.2. For any $\lambda \in\left(\frac{1-3 \eta}{2}, \frac{1-2 \eta}{2}\right), \varepsilon>0$ there exists $\rho_{0}>0$ such that for any $\rho \in\left(0, \rho_{0}\right)$, any minimizer of $E_{\mu}^{\lambda}$ contains points $p, q$ such that

$$
\max \{|p-(-L, h)|,|q-(L, h)|\}<\varepsilon .
$$

Proof. Choose an arbitrary $\lambda \in\left(\frac{1-3 \eta}{2}, \frac{1-2 \eta}{2}\right)$. Let $\mu_{0}$ be the measure defined in $(6)$, and let $\Sigma$ be an arbitrary minimizer of $E_{\mu_{0}}^{\lambda}$.

- Claim: any minimizer $\Sigma \in \operatorname{argmin} E_{\mu_{0}}^{\lambda}$ contains $\{( \pm L, h)\}$.

Choose an arbitrary point $p^{\prime} \in \operatorname{argmin}_{z \in \Sigma}|z-(-L, h)|$, and consider the competitor

$$
\tilde{\Sigma}:=\Sigma \cup \llbracket p^{\prime},(-L, h) \rrbracket \text {. }
$$

By construction

$$
F_{\mu_{0}}(\Sigma)-F_{\mu_{0}}(\tilde{\Sigma}) \geq\left|p^{\prime}-(-L, h)\right| \frac{1-\eta}{2}, \quad \mathcal{H}^{1}(\tilde{\Sigma}) \leq \mathcal{H}^{1}(\Sigma)+\left|p^{\prime}-(-L, h)\right| .
$$

The minimality of $\Sigma$ implies

$$
\frac{1-\eta}{2}\left|p^{\prime}-(-L, h)\right| \leq \lambda\left|p^{\prime}-(-L, h)\right|,
$$

and since $\lambda<(1-\eta) / 2$, it follows $\left|p^{\prime}-(-L, h)\right|=0$. Thus the claim is proven.

Assume (for the sake of contradiction) that the thesis is false, i.e. there exists $\varepsilon>0$ and a sequence $\left\{\rho_{n}\right\} \rightarrow 0$ such that for any $n$ there exists a minimizer $\Sigma_{n} \in \operatorname{argmin} E_{\mu_{\rho_{n}}}^{\lambda}$ satisfying $d\left((-L, h), \Sigma_{n}\right) \geq \varepsilon$. 
Since $\left\{\mu_{\rho_{n}}\right\}^{*} \mu_{0}$ as $n \rightarrow \infty$, there exists $\Sigma_{\infty} \in \operatorname{argmin} E_{\mu_{0}}^{\lambda}$ such that it holds (upon subsequence, which will not be relabeled) $\left\{\Sigma_{n}\right\} \stackrel{d_{\mathcal{H}}}{\rightarrow} \Sigma_{\infty}$. Thus we have

- $d\left((-L, 0), \Sigma_{n}\right) \geq \varepsilon$ for any $n$,

- $(-L, 0) \in \Sigma_{\infty}$,

- $\left\{\Sigma_{n}\right\} \stackrel{d_{\mathcal{H}}}{\rightarrow} \Sigma_{\infty}$,

which gives a contradiction. The proof for $(L, h)$ is analogous.

Lemma 3.3. For any $\lambda \in\left(\frac{1-3 \eta}{2}, \frac{1-2 \eta}{2}\right)$ there exists $\rho_{0}>0$ such that for any $\rho \in\left(0, \rho_{0}\right)$, any minimizer of $E_{\mu}^{\lambda}$ is contained in the half-plane $\{y>0\}$.

Proof. Using Lemmas 3.1 and 3.2 gives the existence of $\rho_{0}>0$ such that for any $\rho \in\left(0, \rho_{0}\right)$ and $\lambda \in\left(\frac{1-3 \eta}{2}, \frac{1-2 \eta}{2}\right)$, any minimizer $\Sigma \in \operatorname{argmin} E_{\mu_{\rho}}^{\lambda}$ satisfies:

- $\Sigma$ is a simple curve with positive length,

- upon reducing the value of $\rho_{0}, \Sigma$ contains points $p, q$ with

$$
|p-(-L, h)| \leq h / 4, \quad|q-(L, h)| \leq h / 4 \text {. }
$$

Choose an arbitrary minimizer $\Sigma$. Let $f:[0,1] \longrightarrow \Sigma$ be a constant speed bijective parameterization, and let $t_{p}:=f^{-1}(p), t_{q}:=f^{-1}(q)$. Since the mass projecting on each endpoint (i.e. $f(0)$ and $f(1))$ is at least $\lambda$, the mass projecting on $f((0,1))$ is at most $1-2 \lambda$. Moreover, the existence of $p$ implies that any point $z \in B((-L, h), \rho)$ satisfies $|z-p| \leq 2 \rho+h / 4$. Since at least $\lambda$ mass projects on $f(0)$, this forces (upon using parameterization $g:[0,1] \longrightarrow \Sigma, g(t):=f(1-t)$ instead of $f$ ) $d(f(0),(-L, h)) \leq h / 4+\rho$, thus (upon further imposing $\rho_{0} \leq h / 24$ )

$$
d(f(0),(-L, h)) \leq h / 4+\rho \leq h / 3 .
$$

Analogously $d(f(1),(L, h)) \leq h / 3$. In particular $f(0)$ and $f(1)$ belong to the half-plane $\{y \geq$ $2 h / 3\}$.

If $f(0,1)$ contains a point $v=f(T) \in\{y=0\}$, then

$$
\left\|f^{\prime}\right\|_{T V} \geq \pi-f(0) \widehat{f(T)} f(1) .
$$

Combining with conditions

$$
d(f(0),(-L, h)) \leq h / 3, \quad d(f(1),(L, h)) \leq h / 3,
$$

elementary geometry gives that the amplitude of angle $f(0) \widehat{f(T)} f(1)$ is bounded from above by the amplitude of angle $\widehat{p_{-p p_{+}}}$where

$$
p_{-}:=\left(-L-\frac{h}{3}, \frac{2}{3} h\right), \quad p:=(0,0), \quad p_{+}:=\left(+L+\frac{h}{3}, \frac{2}{3} h\right) .
$$

Direct computation gives

thus

$$
\widehat{p_{-p p_{+}}}=2 \arctan \frac{L+h / 3}{2 h / 3}
$$

$$
\left\|f^{\prime}\right\|_{T V((0,1))} \geq \pi-f(0) \widehat{f(T)} f(1) \geq \pi-2 \arctan \frac{L+h / 3}{2 h / 3} .
$$


Proposition 2.6 gives

$$
\left\|f^{\prime}\right\|_{T V((0,1))} \leq \frac{\pi}{2 \lambda}(1-2 \lambda)
$$

thus a necessary condition (for $\Sigma \cap\{y=0\} \neq \emptyset$ ) is

$$
\pi-2 \arctan \frac{L+h / 3}{2 h / 3} \leq \frac{\pi}{2 \lambda}(1-2 \lambda) .
$$

This is a contradiction for any $\lambda \in\left(\frac{1-3 \eta}{2}, \frac{1-2 \eta}{2}\right)$, since the left hand side term is roughly comparable with $\frac{4}{3 L}$, while the right hand side term is roughly comparable with $\pi \eta \ll 1 / L$. As both $f(0)$ and $f(1)$ are contained in the half-plane $\{y>0\}$, this ensures that the entire set $f([0,1])=\Sigma$ is contained in the half-plane $\{y>0\}$.

Note that the same argument proves that there exists $\lambda_{0}<\frac{1-2 \eta}{2}$ such that:

- for any $\lambda \in\left(\lambda_{0}, \frac{1-2 \eta}{2}\right)$, there exists $\rho_{0}>0$ such that for any $\rho \in\left(0, \rho_{0}\right)$, any minimizer of $E_{\mu_{\rho}}^{\lambda}$ (here we highlighted the dependency on $\rho$ ) is contained in the half-plane $\{y>h / 10\}$.

The same argument also proves that for such $\lambda, \rho$, any minimizer is contained in the half-plane $\{y<2 h\}$. Thus we have proven:

Lemma 3.4. There exist $\rho, \lambda<\frac{1-2 \eta}{2}$ such that any minimizer of $E_{\mu}^{\lambda}$ is contained in the strip $\{h / 10<$ $y<2 h\}$. Thus any point of $\operatorname{supp}\left(\mu_{\text {light }}\right)$ has lower $y$ coordinate than points of $\Sigma$.

Note that (in view of Lemma 3.2 and for suitable choice of $\rho$ ) since $L$ has been chosen sufficiently large, the mass supported in $B((-L, h), \rho)$ cannot project on any point $z \in \Sigma \cap\{-10<$ $x<10\}$, since for any $x \in B((-L, h), \rho), z \in\{-10<x<10\}$ it holds $|x-z| \geq L-h-10$, while $|x-p| \leq \rho+h / 4$. The same argument proves that the mass supported in $B((L, h), \rho)$ cannot project to any point in $\{-10<x<10\}$.

Until now we have proven (for suitable choice of parameters):

- for any minimizer $\Sigma$, any point in $B((-L, h), \rho) \cup B((L, h), \rho)$ cannot project on $\Sigma \cap\{-10<$ $x<10\}$

- any minimizer contains points $p, q$ satisfying

$$
|p-(-L, h)| \leq h / 4, \quad|q-(L, h)| \leq h / 4,
$$

- any minimizer is contained in the strip $\{h / 10<y<2 h\}$.

Combining these facts, only the mass supported in $\operatorname{supp}\left(\mu_{\text {light }}\right)$ is projected on $\Sigma \cap\{-10<$ $x<10\}$. Recall that by construction the total mass in $\operatorname{supp}\left(\mu_{\text {light }}\right)$ is $\eta$. Choose parameters $\rho \ll 1, \lambda \in\left(\frac{1-3 \eta}{2}, \frac{1-2 \eta}{2}\right)$ such that the conclusions of Lemmas 3.1, 3.2, 3.3 and 3.4 hold. Note that after fixing $\rho$, the measure $\mu$ is uniquely determined. From now, and for all future reference, the measure $\mu$ and $\lambda$ are fixed.

Note that in our construction, there are values which are "large" (e.g. $m, L$ ), and values which are "small" (e.g. $\rho, 1-2 \lambda, \eta, h / L$ ). In particular the value $h / L$ will often appear as angle. The next definition is useful. 
Definition 3.5. Let $v_{1}, v_{2}$ be non zero vectors of $\mathbb{R}^{2}$. The "angle between" $v_{1}$ and $v_{2}$, which we will denote by $\angle v_{1} v_{2}$, is defined as

$$
\angle v_{1} v_{2}:=\arccos \frac{\left\langle v_{1}, v_{2}\right\rangle}{\left|v_{1}\right|\left|v_{2}\right|} \in[0, \pi]
$$

where $\langle$,$\rangle denotes the standard Euclidean scalar product of \mathbb{R}^{2}$. Given segments/half-lines/lines $l_{1}$ and $l_{2}$, the "angle between $l_{1}$ and $l_{2}$ " (which we denote by $\angle l_{1} l_{2}$ ) is defined as

$$
\min _{\substack{v_{1}\left\|l_{1}, v_{2}\right\| l_{2},\left|v_{1}\right|=\left|v_{2}\right|=1}} \angle v_{1} v_{2} .
$$

For the sake of simplicity, we will say that two segments/half-lines/lines are:

- "almost parallel" is the angle between them has form $c_{1} h / L$, with $-10<c_{1}<10$.

- "almost orthogonal" is the angle between them has form $\pi / 2+c_{1} h / L$, with $-10<c_{1}<10$.

The parameter $\rho$ will have little importance in the following, as its "role" is to ensure that minimizers contain points "close to" $( \pm L, h)$ (i.e. $p$ and $q$ from Lemma 3.2). In the following, it will be clear that corners will arise due to measure $\mu_{\text {light. }}$. Since $\operatorname{supp}\left(\mu_{\text {light }}\right)$ is contained in a narrow strip near $\{x=0\}$, we will tacitly assume (unless explicitly stated) we will work only in $\{-10<x<10\}$, and all statements will tacitly assume that entities involved are contained in $\{-10<x<10\}$.

3.2. Discrete measures. The first step involves approximating $\mu$ with discrete measures. Similarly to [12], given three points $v_{1}, v_{2}, v_{3}$, define the "wedge" $V\left(v_{2}\right)$ as follows:

(1) if $v_{1}, v_{2}, v_{3}$ are collinear, then $V\left(v_{2}\right)$ is the unique line passing through $v_{2}$ and orthogonal to $v_{3}-v_{2}$,

(2) otherwise, let $\theta_{i}:=\frac{v_{i+1}-v_{i}}{\left|v_{i+1}-v_{i}\right|}(i=1,2), \xi:=\frac{\theta_{2}+\theta_{1}}{\left|\theta_{2}+\theta_{1}\right|}, b:=\frac{\theta_{2}-\theta_{1}}{\left|\theta_{2}-\theta_{1}\right|}, \beta:=\mathrm{TA}\left(v_{2}\right) / 2$, and

$$
V\left(v_{2}\right):=v_{2}+\left\{x \in \mathbb{R}^{2}:|\langle\xi, x\rangle| \leq\langle b, x\rangle \tan \beta\right\}
$$

where $\langle$,$\rangle denotes the standard Euclidean scalar product of \mathbb{R}^{2}$.

Note that if $\operatorname{TA}(v)>0$, by definition the wedge $V(v)$ is itself an angle (intended as part of the plane contained between two half-lines starting at the same point). Thus expressions like "bisector of $V(v)$ ", "amplitude of $V(v)$ ", etc. will be used. Note also that its border $\partial V(v)$ is union of two half-lines; while $\partial V(v)$ will play an important role in many proofs, it is rarely important to "distinguish" the half-lines forming it, thus in the following we will often use expressions like " $\partial V(v)$ is union of two half-lines $l_{ \pm}$", without stating precisely which half-line corresponds to $l_{-}$ (nor which half-line corresponds to $l_{+}$).

Let

$$
\begin{aligned}
\mu_{j} & :=\sum_{i} \frac{1-\eta}{2 \cdot \sharp\left(B((-L, h), \rho) \cap \frac{\mathfrak{r}_{n}}{j} \mathbb{Z}^{2}\right)} \delta_{q_{i}^{j}}+\sum_{i} \frac{1-\eta}{2 \cdot \sharp\left(B((L, h), \rho) \cap \frac{\mathfrak{r}_{n}}{j} \mathbb{Z}^{2}\right)} \delta_{\tilde{q}_{i}^{j}} \\
& +\sum_{n=1}^{\infty} \sum_{i} \frac{\mathfrak{m}_{n}}{\sharp\left(\mathfrak{B}_{n} \cap \frac{\mathfrak{r}_{n}}{j} \mathbb{Z}^{2}\right)} \delta_{p_{i, n}^{j}},
\end{aligned}
$$


where $\left\{p_{i, n}^{j}\right\}$ (resp. $\left\{q_{i}^{j}\right\},\left\{\tilde{q}_{i}^{j}\right\}$ ) are the (finitely many) points of the lattice $\mathfrak{B}_{n} \cap \frac{\mathfrak{r}_{n}}{j} \mathbb{Z}$ (resp. $\left.B((-L, h), \rho) \cap \frac{1}{j} \mathbb{Z}^{2}, B((L, h), \rho) \cap \frac{1}{j} \mathbb{Z}^{2}\right)$. Intuitively, the mass supported in $\mathfrak{B}_{n}$ (resp. $B((-L, h), \rho)$, $B((L, h), \rho)$ ) is being uniformly distributed on the (uniform) lattice $\mathfrak{B}_{n} \cap \frac{\mathfrak{r}_{n}}{j} \mathbb{Z}^{2}$ (resp. $B((-L, h), \rho) \cap$ $\left.\frac{1}{j} \mathbb{Z}^{2}, B((L, h), \rho) \cap \frac{1}{j} \mathbb{Z}^{2}\right)$.

We will first work with discrete measures $\mu_{j}$, then take the limit $j \rightarrow \infty$. For future reference, any measure $\mu_{j}$ will refer to the (family of) measures defined in (8). Recall that $\mu$ and $\lambda$ were fixed towards the end of subsection 3.1 .

The first result is an analogous of Lemma 3.4 for minimizers of $E_{\mu_{j}}^{\lambda}$ :

Lemma 3.6. For any index $j$, any minimizer of $E_{\mu_{j}}^{\lambda}$ is contained in the strip $\{h / 10<y<2 h\}$.

Proof. The same argument used in the proof of Lemma 3.4 can be applied without any modification to minimizers of $E_{\mu_{j}}^{\lambda}$.

The next result proves that only corners can talk to positive mass.

Lemma 3.7. For any index $j$, minimizer $\Sigma \in \operatorname{argmin} E_{\mu_{j}}^{\lambda}$, if a point $v \in \Sigma$ satisfies $T M\left(\mu_{j}, v, \Sigma\right)>0$, then $T A(v)>0$. In particular $v$ is a corner.

Proof. Note that Lemma 3.6 implies $\Sigma \subseteq\{y \geq h / 10\}$, while $\operatorname{supp}\left(\mu_{\text {light }}\right) \subseteq\{y \leq h / 100\}$.

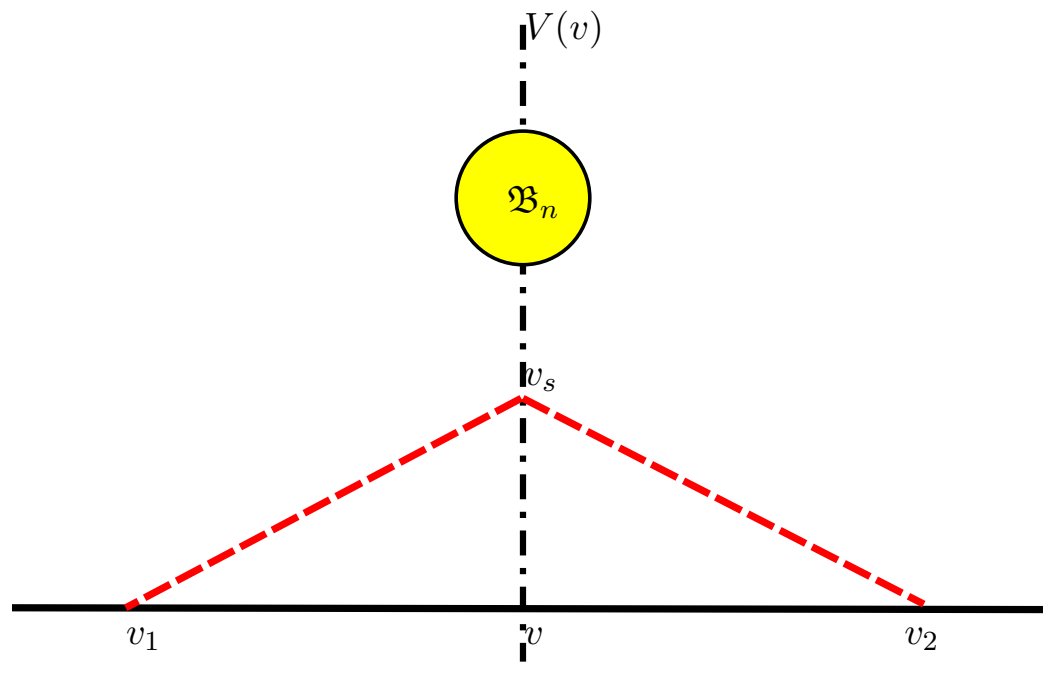

FIGURE 2. This is a schematic representation of the variation. Here $\left(v_{1}-\right.$ $\left.v_{2}\right) \perp\left(v_{s}-v\right)$, and $\left|v_{s}-v\right|=s$.

Assume for the sake of contradiction $\operatorname{TA}(v)=0$, thus $V(v)$ is a line. Hypothesis $T M\left(\mu_{j}, v, \Sigma\right)>$ 0 implies the existence of an index $n$ such that $v$ receives mass from $\mathfrak{B}_{n}$. Let

$$
\Sigma_{s}:=\left(\Sigma \backslash \llbracket v_{1}, v_{2} \rrbracket\right) \cup\left(\llbracket v_{1}, v_{s} \rrbracket \cup \llbracket v_{2}, v_{s} \rrbracket\right) .
$$


Then the same argument from Lemma 3.3 of [9] holds: indeed for $s \ll 1$ it holds $F_{\mu_{j}}\left(\Sigma_{s}\right) \leq$ $F_{\mu_{j}}(\Sigma)-s T M\left(\mu_{j}, v, \Sigma\right), \mathcal{H}^{1}\left(\Sigma_{s}\right)-\mathcal{H}^{1}(\Sigma) \approx O\left(s^{2}\right)$, thus the minimality of $\Sigma$ is contradicted.

The next result is a simple geometric observation, which will be very useful in the following.

Lemma 3.8. Let $p, p^{\prime}, p^{\prime \prime}$ be a triple of points satisfying:

(1) $p^{\prime}, p^{\prime \prime} \in\{y=0\}, p \in\{0<y \leq 2 h\}$ ，

(2) $\widehat{p^{\prime} p p^{\prime \prime}}=2 \theta$, where $\theta \ll 1$ is a given parameter,

(3) $\angle \beta\{x=0\}=\tilde{\tau}$, where $\beta$ denotes the bisector of $\left.\widehat{p^{\prime} p p^{\prime \prime}}\right)$ and $\tilde{\tau} \ll 1$ is a given parameter.

Then it holds

$$
\left|p^{\prime}-p^{\prime \prime}\right| \leq \frac{4 h \sin \theta}{\sin (\tau-\theta) \sin \tau}, \quad \tau:=\frac{\pi}{2}-\tilde{\tau} .
$$

Note that since $|\tau-\pi / 2| \ll 1,|\theta| \ll 1$, this gives

$$
\left|p^{\prime}-p^{\prime \prime}\right| \leq 5 h \theta \text {. }
$$

Proof. Assume without loss of generality $p \in\{x=0\}$. Simple geometric considerations give that $\left|p^{\prime}-p^{\prime \prime}\right|$ is maximized (see Figure 3) when $p \in\{y=2 h\}$.

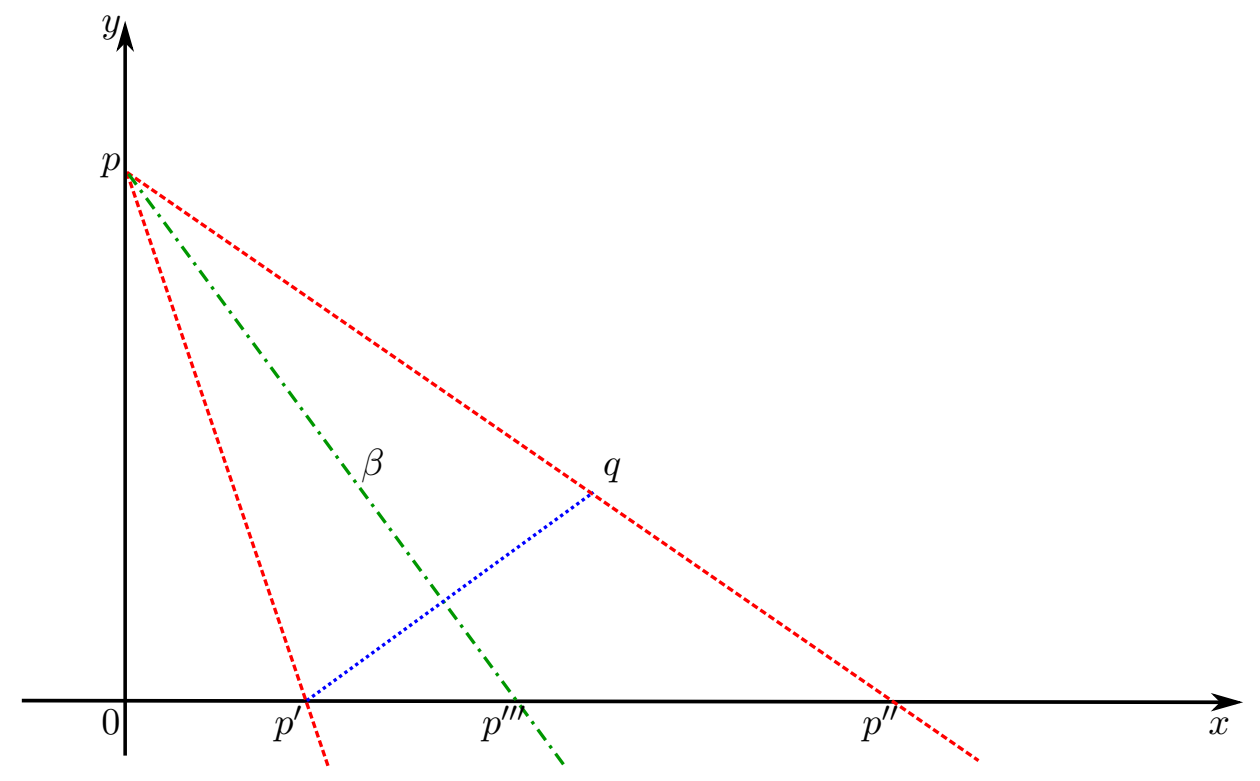

FIGURE 3. This is a schematic representation of the configuration if $\tilde{\tau} \geq \theta$. The proof for case $\tilde{\tau} \leq \theta$ is identical.

Let $q \in \llbracket p, p^{\prime \prime} \rrbracket$ satisfying $\left|p-p^{\prime}\right|=|p-q|$, and denote by $p^{\prime \prime \prime}$ the intersection $\llbracket p, p^{\prime} \rrbracket \cap \beta$. Direct computation gives:

$$
\widehat{q p^{\prime} p}=\pi / 2-\theta, \quad \widehat{p p^{\prime \prime \prime} p^{\prime}}=\frac{\pi}{2}-\tilde{\tau}=\tau, \quad \widehat{p^{\prime} q p^{\prime \prime}}=\pi / 2+\theta, \quad \widehat{p^{\prime \prime} p^{\prime} p}=\pi-\tau-\theta,
$$




$$
\left|p-p^{\prime \prime \prime}\right|=\frac{2 h}{\sin \tau}, \quad\left|p^{\prime}-q\right|=2\left|p-p^{\prime \prime \prime}\right| \sin \tau, \quad \frac{\left|p^{\prime}-p^{\prime \prime}\right|}{\sin (\pi / 2+\theta)}=\frac{\left|p^{\prime}-q\right|}{\sin (\tau-\theta)}
$$

thus

concluding the proof.

$$
\left|p^{\prime}-p^{\prime \prime}\right| \leq \frac{\left|p^{\prime}-q\right|}{\sin (\tau-\theta)}=\frac{2 \sin \theta}{\sin (\tau-\theta)} \frac{2 h}{\sin \tau}
$$

Lemma 3.9. For any index $j$, minimizer $\Sigma \in \operatorname{argmin} E_{\mu_{j}}^{\lambda}$ and corner $v \in \Sigma$, it holds $V(v) \cap \Sigma=\{v\}$. Proof. Note that since any point of $\Sigma \cap\{-10 \leq x \leq 10\}$ can receive mass only from $\bigcup_{n=1}^{\infty} \mathfrak{B}_{n}$, and $\mu_{j}\left(\bigcup_{n=1}^{\infty} \mathfrak{B}_{n}\right)=\eta \ll 1$. It follows $\operatorname{TA}(v) \leq \frac{\pi}{2 \lambda} \eta$. Thus any half-line in $V(v)$ is almost orthogonal to $\{y=0\}$, and any point $w \in(V(v) \cap \Sigma) \backslash\{v\}$ would imply that the curvature of $\Sigma$ is at least $\pi / 4$. Lemma 2.7 gives that the curvature of $\Sigma$ is bounded from above by $\frac{\pi}{2 \lambda}(1-2 \lambda)$, thus such a point $w$ cannot exist.

Lemma 3.10. Let $j$ be a given index, and $\Sigma \in \operatorname{argmin} E_{\mu_{j}}^{\lambda}$ a given minimizer. Let $v_{1}, v_{2} \in \Sigma$ be corners such that $v_{i}$ receives mass from $\mathfrak{B}_{n_{i}}(i=1,2)$ with $n_{1}>n_{2}$. Then $x_{v_{1}}<x_{v_{2}}$, where $x_{p}$ denotes the $x$ coordinate of the point $p$.

Proof. Let $j$ be an arbitrary index, and $\Sigma$ a minimizer. Let $f:[0,1] \longrightarrow \Sigma$ be a constant speed bijective parameterization. Assume the thesis does not hold, i.e. there exist corners $v_{1}, v_{2} \in \Sigma$ such that $v_{i}$ receives mass from $\mathfrak{B}_{n_{i}}(i=1,2)$ with $n_{1}>n_{2}$ and $x_{v_{1}} \leq x_{v_{2}}$. This implies the existence of points $z_{1} \in \mathfrak{B}_{n_{1}}, z_{2} \in \mathfrak{B}_{n_{2}}$ (thus hypothesis $n_{1}>n_{2}$ gives $x_{z_{2}}>x_{z_{1}}$ ), such that $d\left(z_{i}, \Sigma\right)=\left|z_{i}-v_{i}\right|, i=1,2$.

Case $x_{v_{1}}=x_{v_{2}}$. Curvature considerations give

$$
\left\|f^{\prime}\right\|_{T V} \geq \frac{\pi}{3}>\frac{\pi}{2 \lambda}(1-2 \lambda)
$$

which is a contradiction in view of Proposition 2.6

Case $x_{v_{1}}>x_{v_{2}}$. Denote by $y_{p}$ the $y$ coordinate of the point $p$. Note that curvature bounds (Lemma 2.7) impose $\sharp\left(\left\{x=x_{0}\right\} \cap \Sigma\right)=1$ for any $x_{0} \in[-10,10]$. Clearly $\llbracket z_{1}, v_{1} \rrbracket \cap \Sigma=\left\{v_{1}\right\}$ and $\llbracket z_{2}, v_{2} \rrbracket \cap \Sigma=\left\{v_{2}\right\}$. Direct computation on the slope of $L\left(z_{2}, v_{2}\right)$ (defined as the half-line starting in $z_{2}$ and containing $v_{2}$ ) gives

$$
\llbracket z_{1}, v_{1} \rrbracket \cap\left(\llbracket z_{2}, v_{2} \rrbracket \cup\left\{(x, y): x=x_{z_{2}}, y<y_{z_{2}}\right\}\right) \neq \emptyset .
$$

If there exists a point

$$
w^{\prime} \in \llbracket z_{1}, v_{1} \rrbracket \cap\left\{(x, y): x=x_{z_{2}}, y<y_{z_{2}}\right\},
$$

then direct computation (using elementary analytic geometry) gives that the slope of $L\left(z_{1}, w^{\prime}\right)$ (defined as the half-line starting in $z_{1}$ and passing through $\left.w^{\prime}\right)$ forces $L\left(z_{1}, w^{\prime}\right) \cap\left\{x_{z_{1}}<x<\right.$ $10\} \not \supset v_{1}$, which is a contradiction. Thus $\llbracket z_{1}, v_{1} \rrbracket \cap \llbracket z_{2}, v_{2} \rrbracket$ contains a point $w$, and $z_{1}, w, v_{1}$ are collinear. Since $v_{2} \neq v_{1}$, the points $z_{1}, w, v_{2}$ are not collinear: indeed since $\Sigma \cap\{-10 \leq x \leq 10\}$ is finite union of segments, each of which almost parallel to $\{y=0\}$, and any corner $v^{\prime}$ satisfies $T M\left(\mu_{j}, v^{\prime}, \Sigma\right) \leq \eta$, i.e. its wedge $V\left(v^{\prime}\right)$ has amplitude $\mathrm{TA}\left(v^{\prime}\right) \leq \frac{\pi}{2 \lambda} \eta$, and it follows that $\llbracket z_{1}, v_{1} \rrbracket$ 


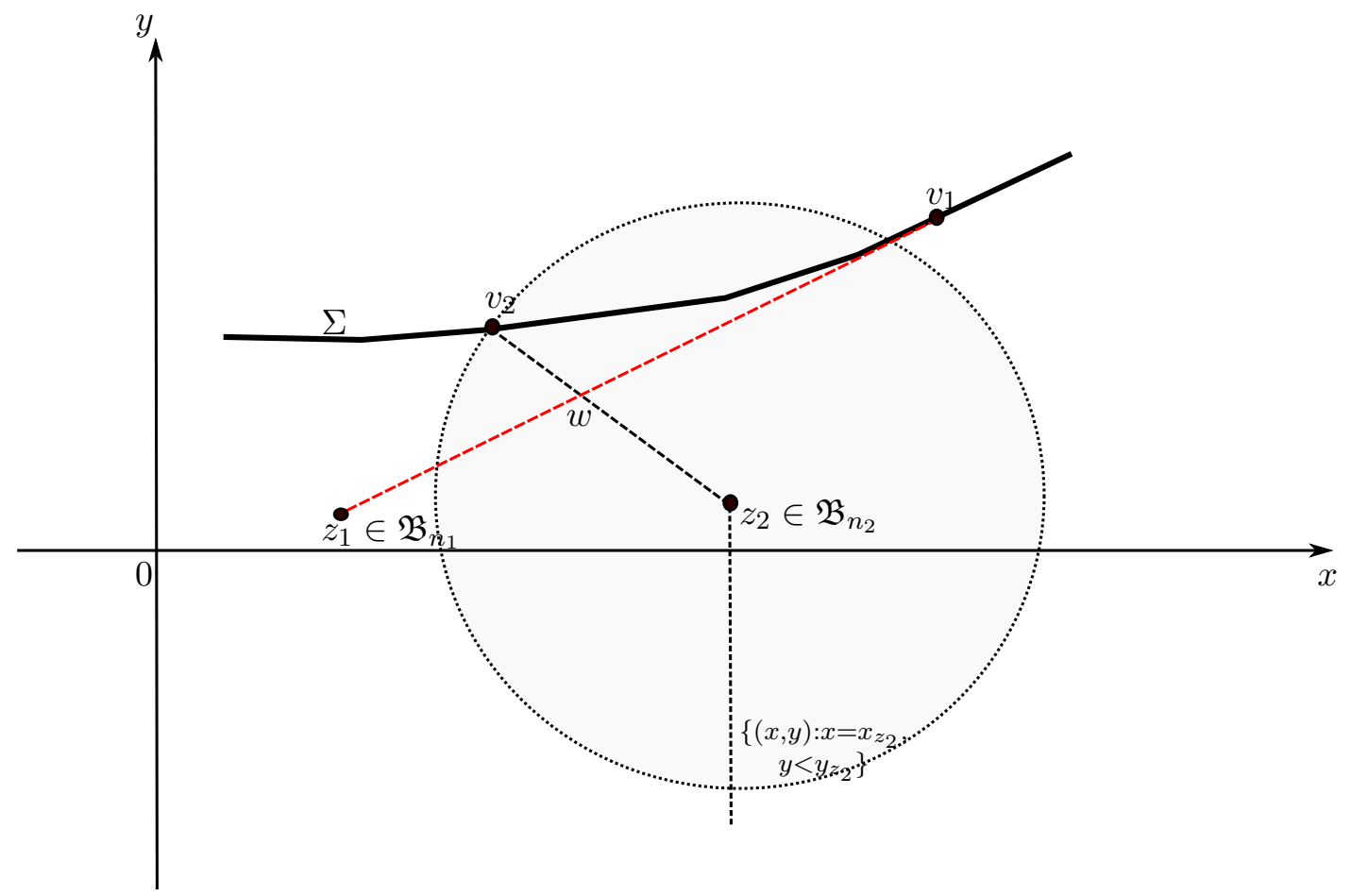

FIGURE 4. This is a schematic representation of the configuration, if contradiction assumption holds. Note that by assumption $v_{1} \notin B\left(z_{2},\left|z_{2}-v_{2}\right|\right)$.

is almost orthogonal to $\{y=0\}$. Thus if $z_{1}, w, v_{2}$ were collinear then the total curvature of $\Sigma$ would exceed $\pi / 4$, which is a contradiction. Thus it follows $\left|z_{1}-v_{2}\right|<\left|z_{1}-w\right|+\left|w-v_{2}\right|$. Since by assumption

$$
\left|z_{2}-w\right|+\left|v_{2}-w\right|=\left|z_{2}-v_{2}\right| \leq\left|z_{2}-v_{1}\right| \leq\left|z_{2}-w\right|+\left|v_{1}-w\right|
$$

it follows $\left|v_{2}-w\right| \leq\left|v_{1}-w\right|$. This in turn gives

$$
\left|z_{1}-v_{2}\right|<\left|z_{1}-w\right|+\left|w-v_{2}\right| \leq\left|z_{1}-w\right|+\left|v_{1}-w\right|=\left|z_{1}-v_{1}\right|,
$$

which is a contradiction.

Lemma 3.11. For any index $j$ and minimizer $\Sigma \in \operatorname{argmin} E_{\mu_{j}}^{\lambda}$, no corner $v \in \Sigma$ satisfies $V(v) \ni(0,0)$.

Proof. Assume (for the sake of contradiction) there exists an index $j$, a minimizer $\Sigma \in \operatorname{argmin} E_{\mu_{j}}^{\lambda}$ and a corner $v \in \Sigma$ satisfying $(0,0) \in V(v)$. Lemma 2.7 forces $v$ to talk to positive mass, and let

$$
n_{0}:=\inf \left\{n: V(v) \cap \mathfrak{B}_{n} \neq \emptyset\right\} .
$$

The $y$ coordinate of $v$ is at most $2 h$ (due to Lemma 3.4), and $\tau:=\angle \beta\{y=0\}$ (here $\beta$ denotes the bisector of $V(v))$ is valued in $\left[\frac{\pi}{2}-\frac{\pi}{2 \lambda}(1-2 \lambda)-\frac{2 h}{L}, \frac{\pi}{2}+\frac{\pi}{2 \lambda}(1-2 \lambda)+\frac{2 h}{L}\right]$ in view of the following facts:

- the curvature of $\Sigma$ does not exceed $\frac{\pi}{2 \lambda}(1-2 \lambda)$ (Lemma 2.7 and Proposition 2.6), 
- $\Sigma \subseteq\{h / 10<y<2 h\}$ (Lemma 3.6), while $\operatorname{supp}\left(\mu_{\text {light }}\right) \subseteq\{y \leq h / 100\}$.

Thus $V(v) \cap\{y=0\}$ contains a point with $x$ coordinate at least $\mathfrak{c}_{n_{0}} / 2$. Let $\theta:=\mathrm{TA}(v) / 2$, Lemma 3.8 gives

$$
\frac{\mathfrak{c}_{n_{0}}}{2} \leq 5 h \sin \theta
$$

i.e.

$$
\theta=\frac{\mathrm{TA}(v)}{2} \geq \sin \theta \geq \frac{1}{5 h} \frac{\mathfrak{c}_{n_{0}}}{2}
$$

By construction, $v$ talks only to masses supported in the union $\bigcup_{n \geq n_{0}} \mathfrak{B}_{n}$, which satisfies

$$
\mu_{j}\left(\bigcup_{n \geq n_{0}} \mathfrak{B}_{n}\right) \leq \sum_{n \geq n_{0}} \mathfrak{m}_{n}
$$

Combining estimate (9), (10) with Lemma 2.7 gives

$$
\frac{1}{5 h} \frac{\mathfrak{c}_{n_{0}}}{2} \leq \theta \leq \frac{\pi}{4 \lambda} \sum_{n \geq n_{0}} \mathfrak{m}_{n}
$$

which is a contradiction.

The next result proves that no corner receives mass from distinct balls $\mathfrak{B}_{n_{1}}, \mathfrak{B}_{n_{2}}, n_{1} \neq n_{2}$.

Lemma 3.12. For index $j$ and minimizer $\Sigma \in \operatorname{argmin} E_{\mu_{j}}^{\lambda}$, there exists no corner $v \in \Sigma$ and indices $n_{1}<n_{2}$ such that the intersections $V(v) \cap \mathfrak{B}_{n_{1}} \neq \emptyset$ and $V(v) \cap \mathfrak{B}_{n_{2}} \neq \emptyset$ are both non empty.

Proof. Assume the opposite, i.e. there exists a corner $v \in \Sigma$ and indices $n_{1}<n_{2}$ such that

$$
V(v) \cap \mathfrak{B}_{n_{1}} \neq \emptyset, V(v) \cap \mathfrak{B}_{n_{2}} \neq \emptyset .
$$

Let

$$
n_{-}:=\inf \left\{n: V(v) \cap \mathfrak{B}_{n} \neq \emptyset\right\}, \quad n_{+}:=\sup \left\{n: V(v) \cap \mathfrak{B}_{n} \neq \emptyset\right\} .
$$

The contradiction assumption ensures $n_{-}<n_{+}$. Note that this gives $\mathcal{L}^{1}(V(v) \cap\{y=0\}) \geq$ $\left(c_{n_{-}}-c_{n_{+}}\right) / 2$. Lemma 3.8 gives

$$
2 h \mathrm{TA}(v) \geq 4 h \sin \frac{\mathrm{TA}(v)}{2} \geq \frac{4}{5} \mathcal{L}^{1}(V(v) \cap\{y=0\}) \geq \frac{2}{5}\left(\mathfrak{c}_{n_{-}}-\mathfrak{c}_{n_{+}}\right) .
$$

However, since by construction only masses supported in $\bigcup_{n=n_{-}}^{n_{+}} \mathfrak{B}_{n}$ can talk to $v$, Lemma 2.7 gives

$$
\mathrm{TA}(v) \leq \frac{\pi}{2 \lambda} \mu_{j}\left(\bigcup_{n=n_{-}}^{n_{+}} \mathfrak{B}_{n}\right)=\frac{\pi}{2 \lambda} \sum_{n=n_{-}}^{n_{+}} \mathfrak{m}_{n}
$$




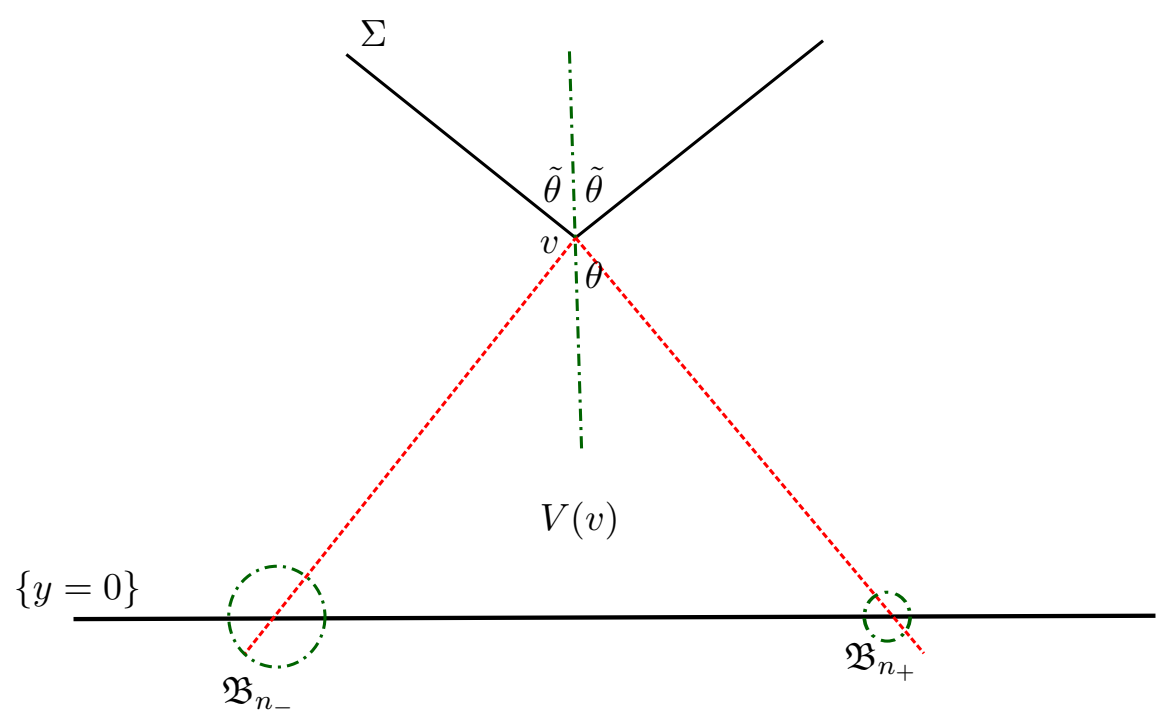

FIGURE 5. If $v$ talks to masses in two distinct balls, $\mathcal{L}^{1}(V(v) \cap\{y=0\})$ is "large", thus the turning angle TA $(v)$ is "large". But there is not enough mass to allow for such large turning angle. Here we omitted representing the balls (if these exist) $\mathfrak{B}_{n}$ with $n_{-}<n<$ $n_{+}$. The relation between $\theta$ and $\tilde{\theta}$ is $\theta=\pi / 2-\tilde{\theta}=(\pi-\mathrm{TA}(v)) / 2$.

thus

$$
\frac{\pi}{\lambda} \sum_{n=n_{-}}^{n_{+}} \mathfrak{m}_{n}=2 h \frac{\pi}{2 \lambda} \sum_{n=n_{-}}^{n_{+}} \mathfrak{m}_{n} \stackrel{12}{\geq} 2 h \mathrm{TA}(v) \stackrel{11}{\geq} \frac{2}{5}\left(\mathfrak{c}_{n_{-}}-\mathfrak{c}_{n_{+}}\right),
$$

which is a contradiction for any $n_{-}$and $n_{+}$.

Combining Lemmas 3.11 and 3.12, we obtain:

- for any index $j$, any minimizer $\Sigma \in \operatorname{argmin} E_{\mu_{j}}^{\lambda}$ contains infinitely many corners.

Consider an index $j$ and a minimizer $\Sigma \in \operatorname{argmin} E_{\mu_{j}}^{\lambda}$ : let $C_{n}$ be the set of corners (of $\Sigma$ ) receiving mass from $\mathfrak{B}_{n}$. Combining Lemmas 3.10 and 3.12 gives:

- for any indices $n_{-}, n_{+}$with $n_{-} \leq n_{+}$, the set $\bigcup_{n=n_{-}}^{n_{+}} C_{n}$ can receive mass only from $\bigcup_{n=n_{-}}^{n_{+}} \mathfrak{B}_{n}$.

Recall that Lemma 3.6 proves that any minimizer $\Sigma \in \operatorname{argmin} E_{\mu_{j}}^{\lambda}$ is contained in the strip $\{h / 10<y<2 h\}$, while all the mass supported within the strip $\{-10<x<10\}$ is contained in the half-plane $\{y \leq h / 100\}$.

The next result proves that given two corners $v_{1} \neq v_{2}$, then their wedges are disjoint.

Lemma 3.13. For any index $j$ and minimizer $\Sigma \in \operatorname{argmin} E_{\mu_{j}}^{\lambda}$, and distinct corners $v_{1}, v_{2} \in \Sigma$, the intersection $V\left(v_{1}\right) \cap V\left(v_{2}\right)$ is empty.

Proof. Note that Lemma 3.6 gives $v_{1}, v_{2} \in \Sigma \subseteq\{y \geq h / 10\}$, while $\operatorname{supp}\left(\mu_{\text {light }}\right) \subseteq\{y \leq h / 100\}$. Then the same argument from Lemma 3.6 of [9] follows. 
The next result estimates the optimal turning angle in relation to the mass projected on a corner. In particular it gives a lower bound estimate.

Lemma 3.14. For any index $j$, minimizer $\Sigma \in \operatorname{argmin} E_{\mu_{j}}^{\lambda}$ and corner $v \in \Sigma$, let $M:=T M\left(\mu_{j}, v, \Sigma\right)$. Then

$$
\mathrm{TA}(v) \rightarrow 0^{+} \Longrightarrow \frac{\mathrm{TA}(v)}{M / \lambda} \rightarrow 1
$$

In particular, if $\mathrm{T} A(v) \leq 0.01$ then

$$
\frac{T A(v)}{M / \lambda} \geq 1 / 2
$$

Proof. Lemmas 3.10, 3.11 and 3.12 give the existence of an unique index $n$ such that $v$ receives mass from $\mathfrak{B}_{n}$. Recall that $v \in \Sigma \subseteq\{y \geq h / 10\}$, while $\mathfrak{B}_{n} \subseteq\{y \leq h / 100\}$. Then the same argument from Lemma 3.4 of [9] follows.

The next result is the core argument of the paper, proving that there exist infinitely many indices $n$ for which there exists a corner $v_{n}$ receiving a positive fraction of the mass supported in $\mathfrak{B}_{n}$.

Lemma 3.15. For any index $j$ and $\Sigma \in \operatorname{argmin} E_{\mu_{j}}^{\lambda}$ it holds:

- there exists an index $n_{0}$ independent of $j$, such that for any index $n \geq n_{0}$ there exists a corner $v_{n} \in \Sigma$ satisfying $\operatorname{TM}\left(\mu_{j}, v_{n}, \Sigma_{j}\right) \geq \mathfrak{m}_{n} / 4$. Moreover, $\operatorname{TA}\left(v_{n}\right) \geq \mathfrak{m}_{n} / 4$.

The proof uses the construction from Lemma 3.7 of [9].

Proof. Fix an index $j$ and a minimizer $\Sigma \in \operatorname{argmin} E_{\mu_{j}}^{\lambda}$. Let $f:[0,1] \longrightarrow \Sigma$ be a constant speed bijective parameterization, consider an index $n$, and let $\left\{v_{i}\right\}_{i=1}^{H}$ be the (finitely many) corners receiving mass from $\mathfrak{B}_{n}$. Recall that by construction $\mu_{j}\left(\mathfrak{B}_{n}\right)=\mathfrak{m}_{n}$.

Let $t_{i}:=f^{-1}\left(v_{i}\right)$ and $M_{i}:=T M\left(\mu_{j}, v_{i}, \Sigma\right)$. Assume

$$
\left(\forall i_{1}, i_{2} \in\{1, \cdots, H\}, i_{1} \neq i_{2}\right) \quad M_{i_{1}}+M_{i_{2}} \leq \mathfrak{m}_{n} / 2 .
$$

The goal is to prove that assumption (13) cannot hold for sufficiently large $n$. Lemma 3.12 implies that any $v_{i}$ talks only to masses in $\mathfrak{B}_{n}$, thus the turning angle $\mathrm{TA}\left(v_{i}\right)$ can be assumed not exceeding $10^{-10}$ since Lemma 2.7 implies TA $\left(v_{i}\right) \leq \frac{\pi}{2 \lambda} \mathfrak{m}_{n}<10^{-10}$. Lemma 3.14 implies

$$
\frac{M_{i}}{2 \lambda} \leq \mathrm{TA}\left(v_{i}\right) \leq \frac{M_{i}}{\lambda}, \quad i=1, \cdots, H
$$

Lemma 3.4 gives $\Sigma \subset\{h / 10<y<2 h\}$, while $\mathfrak{B}_{n} \subseteq\{y<h / 100\}$, thus

$$
d\left(v_{i}, \mathfrak{B}_{n}\right) \geq h / 20, \quad i=1, \cdots, H .
$$

Let $l_{i}^{ \pm}$be the two half-lines which form the border $\partial V\left(v_{i}\right)$ (the exact order is not relevant), and Lemma 3.13 proves that $V\left(v_{i_{1}}\right) \cap V\left(v_{i_{2}}\right)=\emptyset$ whenever $i_{1} \neq i_{2}$.

- Claim: for any index $i$, except at most two, both $l_{i}^{ \pm}$must intersect the border $\partial \mathfrak{B}_{n}$. 


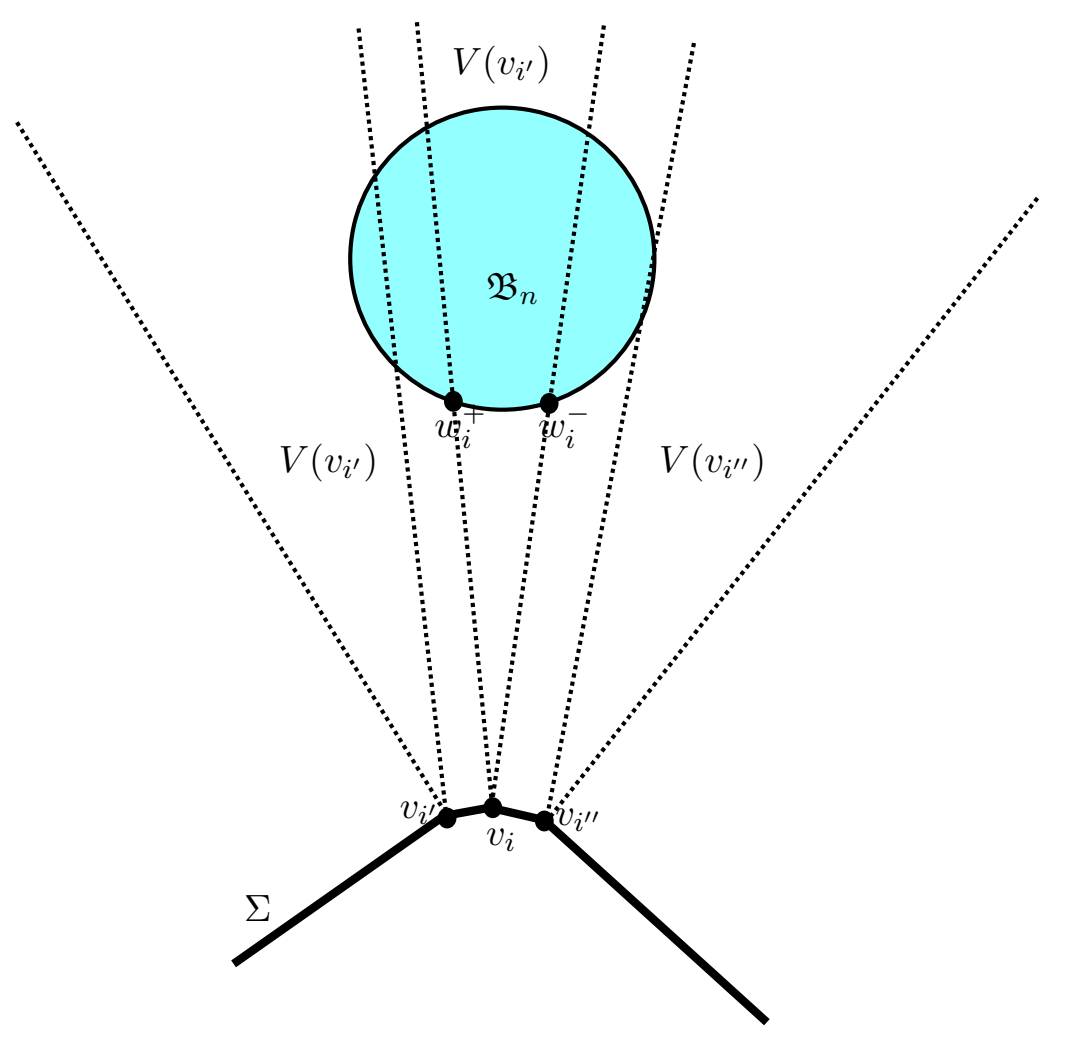

FIGURE 6. A schematic representation of the construction. For the sake of clarity, only three corners (and their wedges) are represented.

This is proven by using the same arguments in the proof of Lemma 3.7 of [9], without any modification.

Using (14), direct computation gives

$$
\min _{z \in l_{i}^{-},\left|z-v_{i}\right| \geq h / 20} d\left(z, l_{i}^{+}\right) \geq \frac{h}{20} \sin \operatorname{TA}\left(v_{i}\right),
$$

thus (since $\mathrm{TA}\left(v_{i}\right) \leq 10^{-10}$ )

$$
\min _{z \in l_{i}^{-},\left|z-v_{i}\right| \geq h / 20} d\left(z, l_{i}^{+}\right) \geq \frac{h}{20} \sin \mathrm{TA}\left(v_{i}\right) \geq \frac{h}{40} \mathrm{TA}\left(v_{i}\right) .
$$

Since for any index $i$, except at most two (which we denote by $i^{\prime}$ and $i^{\prime \prime}$ ), both $l_{i}^{ \pm}$intersect $\partial \mathfrak{B}_{n}$, choose points

$$
w_{i}^{ \pm} \in l_{i}^{ \pm} \cap \partial \mathfrak{B}_{n} \quad i=1, \cdots, H, i \notin\left\{i^{\prime}, i^{\prime \prime}\right\} .
$$


Clearly $V\left(v_{i}\right) \cap \partial \mathfrak{B}_{n}$ contains an arc connecting $w_{i}^{-}$and $w_{i}^{+}$. Thus

$$
\mathcal{H}^{1}\left(V\left(v_{i}\right) \cap \partial \mathfrak{B}_{n}\right) \geq \min _{z \in l_{i}^{-},\left|z-v_{i}\right| \geq h / 20} d\left(z, l_{i}^{+}\right) \stackrel{15}{\geq} \frac{h}{40} \mathrm{TA}\left(v_{i}\right) \stackrel{\text { Lemma } 3.14}{\geq} \frac{h}{40} \frac{M_{i}}{2 \lambda} \stackrel{\lambda<1 / 2}{\geq} \frac{h}{40} M_{i},
$$

Lemma3.13 gives $V\left(v_{i_{1}}\right) \cap V\left(v_{i_{2}}\right)=\emptyset$ whenever $i_{1} \neq i_{2}$. Summing over indices $i \in\{1, \cdots, H\} \backslash\left\{i^{\prime}, i^{\prime \prime}\right\}$ gives

$$
\mathcal{H}^{1}\left(\partial \mathfrak{B}_{n}\right) \geq \sum_{\substack{i=1 \\ i \neq i^{\prime}, i^{\prime \prime}}}^{H} \mathcal{H}^{1}\left(V\left(v_{i}\right) \cap \partial \mathfrak{B}_{n}\right) \stackrel{\sqrt[16]{\geq}}{\geq} \sum_{\substack{i=1 \\ i \neq i^{\prime}, i^{\prime \prime}}}^{H} \frac{h}{40} M_{i} \stackrel{13}{\geq} \frac{h \mathfrak{m}_{n}}{80} .
$$

Let $n_{0}:=\inf \left\{n \in \mathbb{N}: h \mathfrak{m}_{s} / 80>2 \pi \mathfrak{r}_{s}\right.$ for any $\left.s \geq n\right\}$. Clearly (due to the very definition of $\mathfrak{m}_{s}$ and $\mathfrak{r}_{s}$ ) $n_{0}$ is independent of $j$ (explicit computation would give $n_{0}=1$, but this is not relevant for our construction). Thus for any index $n \geq n_{0}$ holds

$$
\sum_{\substack{i=1 \\ i \neq i^{\prime}, i^{\prime \prime}}}^{H} \mathcal{H}^{1}\left(V\left(v_{i}\right) \cap \partial \mathfrak{B}_{n}\right) \stackrel{17}{\geq} \frac{h \mathfrak{m}_{n}}{80}>2 \pi \mathfrak{r}_{n}=\mathcal{H}^{1}\left(\partial \mathfrak{B}_{n}\right),
$$

which is a contradiction.

Thus for any $n \geq n_{0}$ assumption (13) cannot hold, and (for any $n \geq n_{0}$ ) there exist indices $i^{*}, i^{* *}$ such that $M_{i^{*}}+M_{i^{* *}} \geq \mathfrak{m}_{n} / 2$, i.e. $\max \left\{M_{i^{*}}, M_{i^{* *}}\right\} \geq \mathfrak{m}_{n} / 4$. Since $\lambda<1 / 2$, using Lemma 3.14 gives

$$
\max \left\{\operatorname{TA}\left(v_{i^{*}}\right), \operatorname{TA}\left(v_{i^{* *}}\right)\right\} \geq \frac{\max \left\{M_{i^{*}}, M_{i^{* *}}\right\}}{2 \lambda} \geq \frac{\mathfrak{m}_{n}}{4},
$$

and the proof is complete.

3.3. Passing to the limit. Now we have to take the limit $j \rightarrow \infty$. The crucial step is to prove that corners are "far apart". This will be achieved over two lemmas.

Lemma 3.16. For any index $j$ and minimizer $\Sigma \in \operatorname{argmin} E_{\mu_{j}}^{\lambda}$, there exists $n_{0}$ (independent of $j$ ) such that for any corner $v$ talking to some positive mass in $\mathfrak{B}_{n}, n \geq n_{0}$, it holds:

- $V(v) \cap\{y=0\}$ does not contain points $q$ with $\left|x_{q}-\mathfrak{c}_{n}\right|>\mathfrak{c}_{n} / 10$, where $x_{q}$ denotes the $x$ coordinate of $q$.

Proof. If $v$ talks to positive mass supported in $\mathfrak{B}_{n}$, it follows

$$
V(v) \cap \mathfrak{B}_{n} \neq \emptyset \text {. }
$$

Lemma 2.7 gives that the total curvature of $\Sigma$ does not exceed $\frac{\pi}{2 \lambda}(1-2 \lambda) \ll 1$. Combining with Lemmas 3.2 and 3.6 gives that the bisector of $V(v)$ is almost orthogonal to $\{y=0\}$. Since $v$ receives mass only from $\mathfrak{B}_{n}$, Lemma 3.14 implies that the amplitude of $V(v)$ does not exceed $\frac{\pi}{2 \lambda} \mathfrak{m}_{n}$. Elementary geometry proves that $V(v) \cap\{y=0\}$ contains a point $q_{1}$ with $x$ coordinate $x_{q_{1}} \in\left[\mathfrak{c}_{n}-4 \mathfrak{r}_{n}, \mathfrak{c}_{n}+4 \mathfrak{r}_{n}\right]$. Thus Lemma 3.8 implies that for any sufficiently large $n$, the intersection $\mathrm{TA}(v) \cap\{y=0\}$ does not contain points with $x$ coordinate outside $\left[0.9 \mathfrak{c}_{n}, 1.1 \mathfrak{c}_{n}\right]$. 
Lemma 3.17. For any index $j$, minimizer $\Sigma \in \operatorname{argmin} E_{\mu_{j}}^{\lambda}$ and corners $v_{n_{i}}(i=1,2)$ talking to some positive mass supported in $\mathfrak{B}_{n_{i}}(i=1,2)$, it holds:

- there exists a positive constant $C$ independent of $j$ and $\max \left\{n_{1}, n_{2}\right\}$ such that $\left|v_{n_{1}}-v_{n_{2}}\right| \geq C$.

Proof. Assume (by symmetry) $n_{1}<n_{2}$, let $f:[0,1] \longrightarrow \Sigma$ be a constant speed parameterization, and let $t_{n_{i}}:=f^{-1}\left(v_{n_{i}}\right), i=1,2$. Lemma 2.7 gives

$$
\left\|f_{\mid\left[t_{n_{1}}, t_{n_{2}}\right]}^{\prime}\right\|_{T V} \leq \frac{\pi}{2 \lambda} \sum_{n=n_{1}}^{n_{2}} \mathfrak{m}_{n} .
$$

Let $\beta_{i}$ be the bisectors of $V\left(v_{n_{i}}\right), i=1,2$. The angle between $\beta_{1}$ and $\beta_{2}$ is bounded from above by $\frac{\pi}{2 \lambda} \sum_{n=n_{1}}^{n_{2}} \mathfrak{m}_{n}$ since any point $f(t), t \in\left(t_{n_{1}}, t_{n_{2}}\right)$, can only receive mass from $\bigcup_{n=n_{1}}^{n_{2}} \mathfrak{B}_{n}$. Let $q_{i}:=\beta_{i} \cap\{y=0\}$, and if $\beta_{1}$ and $\beta_{2}$ were parallel (note that in general $\beta_{1}$ and $\beta_{2}$ are not parallel), then $\left|v_{n_{1}}-v_{n_{2}}\right| \geq\left|q_{1}-q_{2}\right| / 2$ (since $v_{n_{1}}-v_{n_{2}}$ and $\{y=0\}$ are almost parallel). Since the angle between $\beta_{1}$ and $\beta_{2}$ is at most $\frac{\pi}{2 \lambda} \sum_{n=n_{1}}^{n_{2}} \mathfrak{m}_{n}$, the error (done by assuming $\beta_{1}$ and $\beta_{2}$ are parallel) is at most $\frac{h \pi}{\lambda} \sum_{n=n_{1}}^{n_{2}} \mathfrak{m}_{n} \ll\left|\mathfrak{c}_{n_{1}}-\mathfrak{c}_{n_{2}}\right|$.

By construction $\mathfrak{c}_{n_{2}}<10^{-10} \mathfrak{c}_{n_{1}}$. Denote by $x_{p}$ the $x$ coordinate of the point $s$. Since by definition $q_{i} \in V\left(v_{n_{i}}\right) \cap\{y=0\}(i=1,2)$, Lemma 3.16 gives $\left|x_{q_{i}}-\mathfrak{c}_{n_{i}}\right| \leq \mathfrak{c}_{n_{i}} / 10(i=1,2)$, thus

$$
x_{q_{1}} \geq 0.9 \mathfrak{c}_{n_{1}}, \quad x_{q_{2}} \leq 1.1 \mathfrak{c}_{n_{2}},
$$

i.e. $\left|q_{1}-q_{2}\right| \geq 0.8 \mathfrak{c}_{n_{1}}$. Letting $C:=0.8 \mathfrak{c}_{n_{1}}$ concludes the proof.

Now we can pass to the limit $j \rightarrow \infty$ : for any index $j$ choose a minimizer $\Sigma_{j} \in \operatorname{argmin} E_{\mu_{j}}^{\lambda}$, and let $f_{j}:[0,1] \longrightarrow \Sigma_{j}$ a constant speed bijective parameterization. Since $\left\{\mu_{j}\right\} \stackrel{*}{\rightarrow} \mu$, upon subsequence it holds (using Lemma 2.8) $\left\{f_{j}\right\} \rightarrow f$ uniformly, for some $\Sigma \in \operatorname{argmin} E_{\mu}^{\lambda}$ and parameterization $f:[0,1] \longrightarrow \Sigma$. Thus

$$
\left\{\Sigma_{j}\right\} \stackrel{d_{\mathcal{H}}}{\rightarrow} \Sigma \in \operatorname{argmin} E_{\mu}^{\lambda} .
$$

Lemma 3.15 proves the existence of $n_{0}$ (independent of $j$ ) such that for any $n \geq n_{0}$, each minimizer $\Sigma_{j}$ contains a corner $v_{n}^{j}$ satisfying $\operatorname{TA}\left(v_{n}^{j}\right) \geq \mathfrak{m}_{n} / 4$. In other words, the measure $f_{j}^{\prime \prime}$ has an atom of measure at least $\mathfrak{m}_{n} / 4$ at time $t_{n}^{j}:=f_{j}^{-1}\left(v_{n}^{j}\right)$. Again passing to the limit $j \rightarrow \infty$, it holds (upon subsequence) $\left\{t_{n}^{j}\right\} \rightarrow t_{n}$, thus $f^{\prime \prime}$ has an atom of measure at least $\mathfrak{m}_{n} / 4$ in $t_{n}$. Note that an atom for the measure $f^{\prime \prime}$ corresponds to a jump for the tangent derivative $f^{\prime}$, i.e. a corner for $\Sigma$.

Lemma 3.17ensures that $v_{n_{1}} \neq v_{n_{2}}$ whenever $n_{1} \neq n_{2}$. Thus passing to the limit $j \rightarrow \infty, \Sigma$ has infinitely many corners. Let $v$ be an accumulation point of $\left\{v_{n}\right\}, v \notin\left\{v_{n}\right\}$. It remains to prove that such $v$ is not a corner itself.

Lemma 3.18. Such accumulation point $v$ is not a corner itself.

Before the proof, a preliminary lemma is required.

Lemma 3.19. For any sequence of corners $\left\{v_{s}\right\} \subseteq \Sigma$ (not definitely constant), the sequence $\left\{x_{v_{s}}\right\}$ admits $a$ strictly decreasing subsequence $\left\{x_{v_{g(s)}}\right\}$, where $x_{p}$ denotes the $x$ coordinate of the point $p$. 
Proof. The proof is similar to the proof of Lemma 3.10. Assume (for the sake of contradiction) that there exist indices $n, N$ with $n<N$, distinct corners $v_{n}, v_{N} \in \Sigma$ and points $z_{n} \in \mathfrak{B}_{n}$, $z_{N} \in \mathfrak{B}_{N}$, such that

$$
\left|v_{n}-z_{n}\right|=d\left(z_{n}, \Sigma\right), \quad\left|v_{N}-z_{N}\right|=d\left(z_{N}, \Sigma\right), \quad x_{v_{n}} \leq x_{v_{N}} .
$$

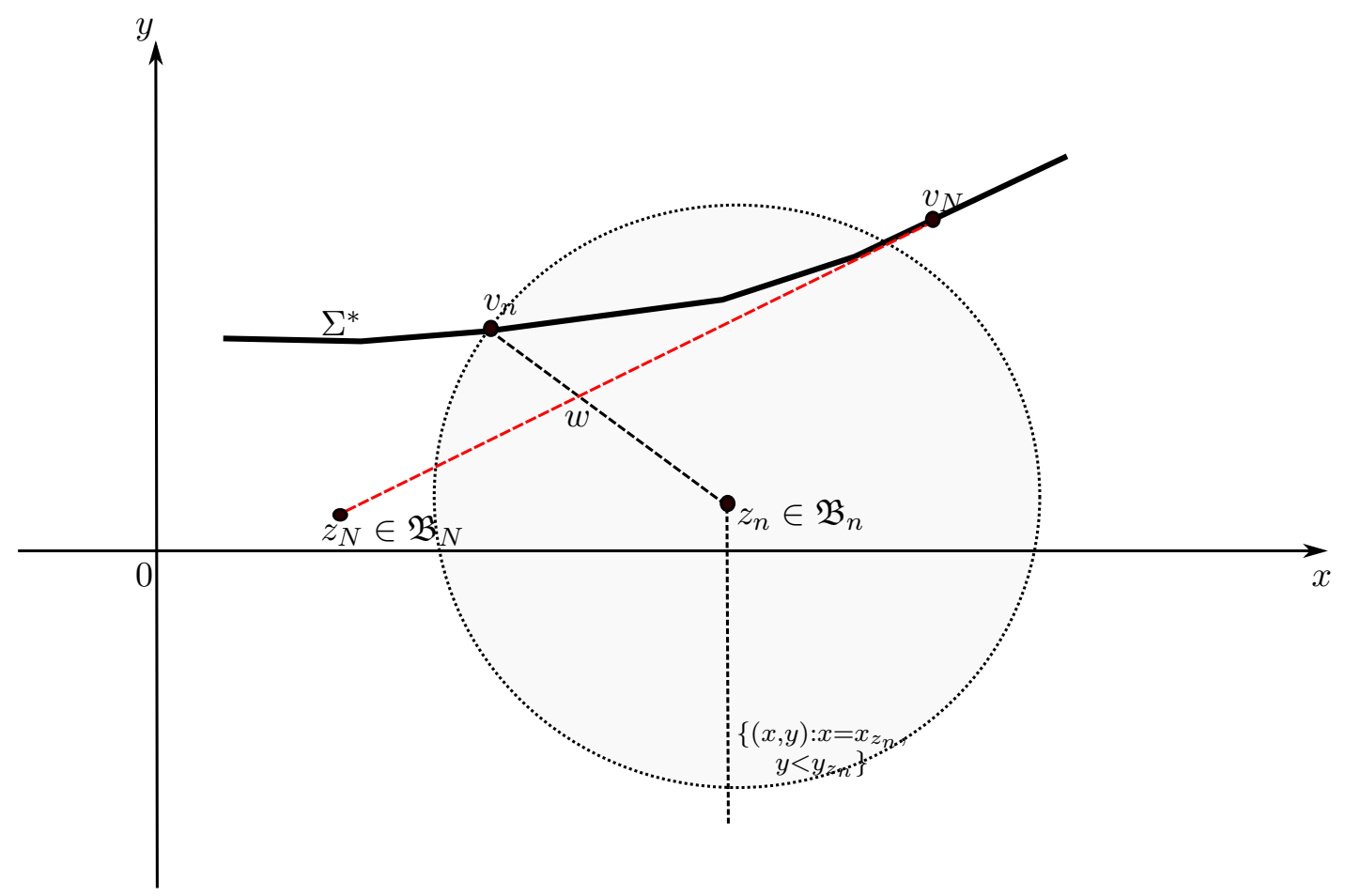

FIGURE 7. This is a schematic representation of the configuration, if contradiction assumption holds. Note that by assumption $v_{N} \notin B\left(z_{n},\left|z_{n}-v_{n}\right|\right)$.

Denote by $y_{p}$ the $y$ coordinate of the point $p$. Let $\Sigma^{*}:=\Sigma \cap\left\{x \leq x_{v_{n}}\right\}$, and note that curvature bounds (Lemma 2.7) impose $\sharp\left(\left\{x=x_{0}\right\} \cap \Sigma\right)=1$ for any $x_{0} \in[-10,10]$, and $\llbracket z_{n}, v_{n} \rrbracket \cap \Sigma=\left\{v_{n}\right\}$. Case $x_{v_{n}}<x_{v_{N}}$ remains. Clearly

$$
\llbracket z_{N}, v_{N} \rrbracket \cap \Sigma^{*}=\emptyset
$$

since the opposite would contradict either $\left|z_{N}-v_{N}\right|=d\left(z_{N}, \Sigma\right)$ or $x_{v_{N}}>x_{v_{n}}$. Similarly to the proof of Lemma 3.10, direct computation of the slope of $L\left(z_{n}, v_{n}\right)$ (defined as the half-line starting in $z_{n}$ and containing $v_{n}$ ) forces

$$
\llbracket z_{N}, v_{N} \rrbracket \cap\left(\llbracket z_{n}, v_{n} \rrbracket \cup\left\{(x, y): x=x_{z_{n}}, y<y_{z_{n}}\right\}\right) \neq \emptyset .
$$

If there exists a point $w^{\prime} \in \llbracket z_{N}, v_{N} \rrbracket \cap\left\{(x, y): x=x_{z_{n}}, y<y_{z_{n}}\right\}$, then direct computation gives that the slope of $L\left(z_{N}, w^{\prime}\right)$ (defined as the half-line starting in $z_{N}$ and passing through $w^{\prime}$ ) satisfies $L\left(z_{N}, w^{\prime}\right) \cap\{-10 \leq x \leq 10\} \not \supset v_{N}$, which is a contradiction. Thus $\llbracket z_{N}, v_{N} \rrbracket \cap \llbracket z_{n}, v_{n} \rrbracket$ 
contains a point $w$, and $z_{N}, w, v_{N}$ are collinear. Since $v_{n} \neq v_{N}$, the points $z_{N}, w, v_{n}$ are not collinear: indeed $\llbracket z_{N}, v_{N} \rrbracket$ is almost orthogonal to $\{y=0\}$, thus if $z_{N}, w, v_{n}$ were collinear, the total curvature of $\Sigma$ would exceed $\pi / 4$, prohibited by Lemma 2.7 . Thus $\left|z_{N}-v_{n}\right|<\left|z_{N}-w\right|+$ $\left|w-v_{n}\right|$. Since by assumption

$$
\left|z_{n}-w\right|+\left|v_{n}-w\right|=\left|z_{n}-v_{n}\right| \leq\left|z_{n}-v_{N}\right| \leq\left|z_{n}-w\right|+\left|v_{N}-w\right|,
$$

it follows $\left|v_{n}-w\right| \leq\left|v_{N}-w\right|$. This in turn gives

$$
\left|z_{N}-v_{n}\right|<\left|z_{N}-w\right|+\left|w-v_{n}\right| \leq\left|z_{N}-w\right|+\left|v_{N}-w\right|=\left|z_{N}-v_{N}\right|
$$

which is a contradiction. Since for any ball $\mathfrak{B}_{s}$ there exists a corner $v_{s}$ talking to positive mass supported on $\mathfrak{B}_{s}$, the proof is complete.

Proof. (of Lemma 3.18) For the sake of brevity, the notation $x_{p}$ (resp. $y_{p}$ ) will denote the $x$ (resp. $y$ ) coordinate of $p$.

Choose a sequence $\left\{v_{s}\right\} \rightarrow v$, and assume (in view of Lemma 3.19 $\left\{x_{v_{s}}\right\}$ strictly decreasing.

Assume (for the sake of contradiction) there exists an index $n$ such that $v$ talks to some point $z \in \mathfrak{B}_{n}$. Choose an index $N>n$, and a corner $v_{N}$ talking to some point $z_{N} \in \mathfrak{B}_{N}$. Such points exist due to our construction. Let $l$ be the line through $v_{N}$ and $z_{N}$, and let $l^{\prime}:=\llbracket v, z \rrbracket$. By construction $l$ is almost orthogonal to $\{y=0\}$. Recall that $\Sigma \subseteq\{h / 10 \leq y \leq 2 h\}$ (Lemma 3.4), while all balls $\mathfrak{B}_{n}$ are contained in $\{y \leq h / 100\}$. By construction $x_{v}<x_{v_{N}}, x_{z_{n}}>x_{z_{N}}$. Since $l$ is almost orthogonal to $\{y=0\}$, and the total curvature of $\Sigma \cap\{-10 \leq x \leq 10\}$ does not exceed $\frac{\pi}{2 \lambda} \eta \ll 1$, this implies the existence of a point $w \in l \cap l^{\prime}$. Let

- $l^{-}:=$be half-line (contained in $l$ ) starting from $z_{N}$ and not containing $v_{N}$,

- $l^{+}:=$be half-line (contained in $l$ ) starting from $v_{N}$ and not containing $z_{N}$,

- $l^{\circ}:=\llbracket z_{N}, v_{N} \rrbracket$.

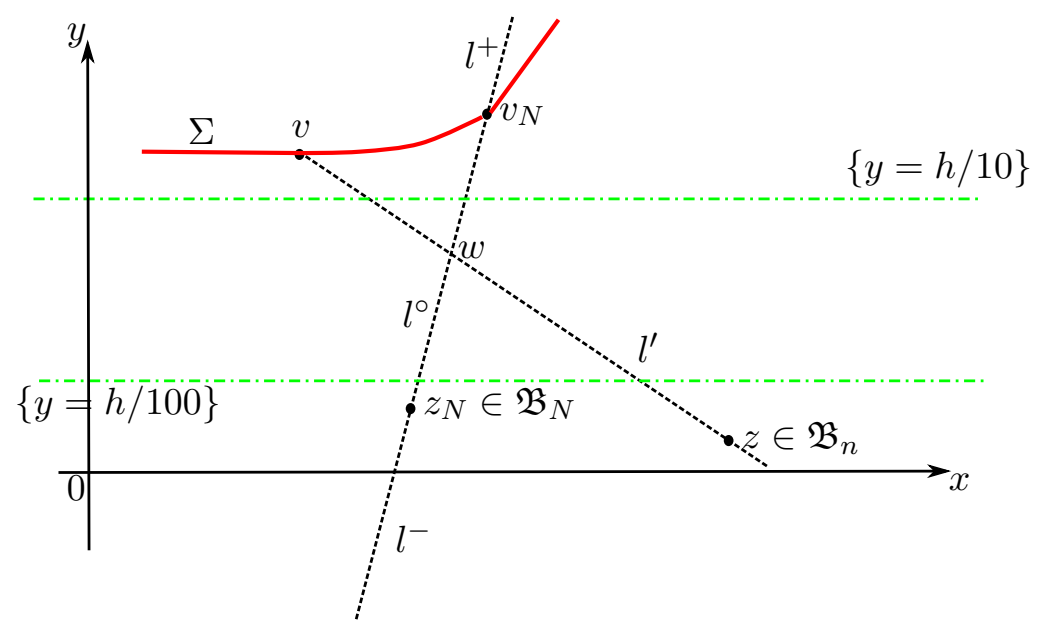

FIGURE 8. This is a schematic representation of the configuration.

The following possibilities arise: 
(1) $w \in l^{-}$: in this case the slope of $L(z, w)$ (defined as the half-line starting in $z$ and passing through $w$ ) prohibits $l^{\prime} \cap\{y>h / 10\} \cap\{-10 \leq x \leq 10\} \ni v$, which is a contradiction,

(2) $w \in l^{+}$: this would give $|z-v| \geq|z-w| \geq\left|z-v_{N}\right|$, with equality holding only if $v=v_{N}$, prohibited by our assumptions,

(3) $w \in \llbracket v_{N}, z_{N} \rrbracket$ : note that $z_{N}, w, v_{N}$ are collinear, while $z_{N}, w, v$ are not: indeed if $z_{N}, w, v$ were collinear, since $\llbracket z_{N}, v_{N} \rrbracket$ is almost orthogonal to $\{y=0\}$, then the total curvature of $\Sigma$ would exceed $\pi / 4$, prohibited by Lemma 2.7. Thus

$$
\left|z_{N}-v\right|<\left|z_{N}-w\right|+|v-w|, \quad\left|z_{N}-v_{N}\right|=\left|z_{N}-w\right|+\left|v_{N}-w\right|,
$$

and $|v-w|>\left|v_{N}-w\right|$. This yields

$$
\left|z-v_{N}\right| \leq|z-w|+\left|v_{N}-w\right|<|z-w|+|v-w|=|z-v|
$$

i.e. $z$ cannot talk to $v$, which is a contradiction.

Thus all three cases lead to a contradiction, i.e. such point $v$ cannot talk to any mass in any ball $\mathfrak{B}_{n}$. Using Lemma 2.7 finally gives $\mathrm{TA}(v)=0$, i.e. $v$ is not a corner.

Thus we have proven:

Theorem 3.20. There exists a measure $\mu$ and a parameter $\lambda$, such that there exists $\Sigma \in \operatorname{argmin} E_{\mu}^{\lambda}$ containing a sequence of corners $\left\{v_{n}\right\}$ satisfying:

- for any $n T A\left(v_{n}\right) \geq \mathfrak{m}_{n} / 4$, i.e. $v_{n}$ is a corner,

- $\left\{v_{n}\right\} \rightarrow v \in \Sigma, T A(v)=0$, i.e. $v$ is not a corner.

Corollary 3.21. The minimizer $\Sigma$ from Theorem 3.20 is also minimizer for the constrained problem

$$
\min _{\mathcal{H}^{1}(\cdot) \leq \mathcal{H}^{1}(\Sigma)} \int_{\mathbb{R}^{2}} d(x, \cdot) d \mu .
$$

Proof. In [2] it has been proven that any minimizer $\tilde{\Sigma}$ of (18) satisfies $\mathcal{H}^{1}(\tilde{\Sigma})=\mathcal{H}^{1}(\Sigma)$, thus if $\Sigma$ is not a minimizer of (18), choosing $\Sigma^{*}$ minimizer of (18) would give

$$
\int_{\mathbb{R}^{2}} d\left(x, \Sigma^{*}\right) d \mu<\int_{\mathbb{R}^{2}} d(x, \Sigma) d \mu, \quad \mathcal{H}^{1}\left(\Sigma^{*}\right)=\mathcal{H}^{1}(\Sigma)
$$

contradicting $\Sigma \in \operatorname{argmin} E_{\mu}^{\lambda}$.

Acknowledgments. The author warmly thanks the Center of Nonlinear Analysis (NSF grant DMS-0635983), where part of this research was carried out, and acknowledges the support by ICTI and FCT (grant UTA_CMU/MAT/0007/2009). He is grateful to Filippo Santambrogio and Dejan Slepčev for useful comments and suggestions, and to Universidade Nova de Lisboa for its hospitality. This research was conducted when the author was postdoctoral associate at Center of Nonlinear Analysis of Carnegie Mellon University. 


\section{REFERENCES}

[1] Buttazzo, G., Mainini, E. and Stepanov, E.: Stationary configurations for the average distance functional and related problems, Control Cybern. 38, 1107-1130 (2009)

[2] Buttazzo, G., Oudet, E., and Stepanov, E.: Optimal transportation problems with free Dirichlet regions , Progr. Nonlinear Differential Equations Appl. 51, 41-65 (2002)

[3] Buttazzo, G. and Stepanov, E.: Minimization problems for average distance functionals, In: Pallara, D. (ed.), Calculus of Variations: Topics from the Mathematical Heritage of Ennio De Giorgi, Quaderni di Matematica, vol. 14, 47-83, Seconda Università di Napoli, Caserta, (2004)

[4] Buttazzo, G. and Stepanov, E.: Optimal transportation networks as free Dirichlet regions for the MongeKantorovich problem, Ann. Sc. Norm. Sup. Pisa Cl. Sci. 2, 631-678 (2003)

[5] Gilbert, E.N. and Pollack, H.O.: Steiner minimal trees, SIAM J. Math. Anal. 16, 1-29 (1968)

[6] Hwang, F.K., Richards, D.S. and Winter, P.: The Steiner tree problem, Ann. of Discr. Math. 53, North-Holland Publishing Co. (1992)

[7] Lemenant, A.: A presentation of the average distance minimizing problem, POMI 390, 117-146 (2011)

[8] Lemenant, A.: About the regularity of average distance minimizers in $\mathbb{R}^{2}$, Preprint (available at http://cvgmt.sns.it/paper/1615/)

[9] Lu, X.Y.: More counterexamples to regularity for minimizers of the average distance problem, Preprint (available at http: / / cvgmt.sns.it/paper/2304/)

[10] Lu, X.Y. and Slepčev, D.: Properties of minimizers of average distance problem via discrete approximation of measures, SIAM J. Math. Anal. 45(5), 3114-3131 (2013)

[11] Paolini, E. and Stepanov, E.: Qualitative properties of maximum and average distance minimizers in $\mathbb{R}^{n}$, J. Math. Sci. (N. Y.) 122(3), 3290-3309 (2004)

[12] Slepčev, D.: Counterexample to regularity in average-distance problem, Ann. Inst. Henri Poincaré (C), 169-184 (2014)

(1) Department of Mathematics, Instituto Superior Técnico, 1 Avenida Rovisco Pais, 1049-001 LisBON, PORTUGAL

(2) Department of Mathematical Sciences, Carnegie Mellon University, Pittsburgh, PA, 15213,United STATES, EMAIL: XINYANG@ANDREW.CMU.EDU 